\title{
Determination of quantum numbers for several excited charmed mesons observed in $B^{-} \rightarrow D^{*+} \pi^{-} \pi^{-}$decays

\author{
R. Aaij et al.*
} \\ (LHCb Collaboration)
}

(Received 15 November 2019; accepted 23 January 2020; published 20 February 2020)

\begin{abstract}
A four-body amplitude analysis of the $B^{-} \rightarrow D^{*+} \pi^{-} \pi^{-}$decay is performed, where fractions and relative phases of the various resonances contributing to the decay are measured. Several quasi-model-independent analyses are performed aimed at searching for the presence of new states and establishing the quantum numbers of previously observed charmed meson resonances. In particular the resonance parameters and quantum numbers are determined for the $D_{1}(2420), D_{1}(2430), D_{0}(2550), D_{1}^{*}(2600), D_{2}(2740)$ and $D_{3}^{*}(2750)$ states. The mixing between the $D_{1}(2420)$ and $D_{1}(2430)$ resonances is studied and the mixing parameters are measured. The dataset corresponds to an integrated luminosity of $4.7 \mathrm{fb}^{-1}$, collected in proton-proton collisions at center-of-mass energies of 7, 8 and $13 \mathrm{TeV}$ with the LHCb detector.
\end{abstract}

DOI: $10.1103 /$ PhysRevD.101.032005

\section{INTRODUCTION}

Charmed-meson spectroscopy provides a powerful test of quark-model predictions in the Standard Model. Many charmed-meson states predicted in the 1980s (see e.g., Ref. [1] and references within, and Ref. [2] for a recent update) have not yet been observed experimentally. The study of $D \pi$ final states enables a search for natural spinparity resonances, $\left[P=(-1)^{J}\right.$, labeled as $\left.D^{*}\right]$, while the study of $D^{*} \pi$ final states provides the possibility of studying both natural and unnatural spin-parity states, except for the $J^{P}=0^{+}$case, which is forbidden due to angular momentum and parity conservation. Above the ground $1 S$ states $\left(D, D^{*}\right)$, two of the orbital $1 P$ excitations, $D_{1}(2420)$ and $D_{2}^{*}(2460)$, are experimentally well established [3] since they have relatively narrow widths $(\sim 30 \mathrm{MeV}) .{ }^{1}$ One of the broad $1 P$ states, $D_{0}^{*}(2400)$, has been studied by the Belle, BABAR and LHCb collaborations in exclusive $B$ decays [4-8]. Another broad $1 P$ state, $D_{1}(2430)$, has been observed by the Belle collaboration in the amplitude analysis of $560 B^{-} \rightarrow D^{*+} \pi^{-} \pi^{-}$decays [4]. The study of the $B \rightarrow D^{* *} l \nu$ decay by the $B A B A R$ [9] and Belle [10] collaborations gives contradicting results on the production of $D_{1}(2430)$ in semileptonic $B$ decays.

The search for excited charmed mesons, labeled $D_{J}$, can be performed using two different approaches: using inclusive reactions, or through amplitude analyses of exclusive

*Full author list given at the end of the article.

${ }^{1}$ The system of units where $c=1$ is adopted throughout.

Published by the American Physical Society under the terms of the Creative Commons Attribution 4.0 International license. Further distribution of this work must maintain attribution to the author(s) and the published article's title, journal citation, and DOI. Funded by SCOAP ${ }^{3}$.
$B$ decays. In inclusive $D^{(*)} \pi$ production, where production of any $J^{P}$ state is permitted, large data samples are obtained, however, in addition to a large combinatorial background. In three-body $D_{J}$ decays it is also possible to perform an angular analysis and therefore distinguish between natural and unnatural spin-parity assignments. The amplitude analysis of $B$ decays, on the other hand, although often with limited data sample size, allows a full spin-parity analysis of the charmed mesons present in the decay. In addition, backgrounds are usually rather low and comparatively well understood.

Using the first approach, the BABAR [11] (in $e^{+} e^{-}$ annihilations) and LHCb [12] (in $p p$ interactions) collaborations, have analyzed the inclusive production of the $D^{+} \pi^{-}, D^{0} \pi^{+}$and $D^{*+} \pi^{-}$final states. Both collaborations observe four resonances, labeled by the Particle Data Group (PDG) as $D_{0}(2550), D_{J}^{*}(2600), D(2740)$ and $D_{3}^{*}(2750)$ [3]. The $D_{0}(2550)$ and $D(2740)$ decay angular distributions are consistent with an unnatural spin-parity, while the $D_{J}^{*}(2600)$ and $D_{3}^{*}(2750)$ states are assigned natural parities. The $D_{3}^{*}(2750)$ resonance was also observed in $B$-decay amplitude analyses of $B^{0} \rightarrow \bar{D}^{0} \pi^{+} \pi^{-}$[13] and $B^{-} \rightarrow D^{+} \pi^{-} \pi^{+}$[14] by the LHCb collaboration, where quantum numbers were determined to be $J^{P}=3^{-}$. For the $D_{0}(2550)$ meson, angular distributions are consistent with a $J^{P}=0^{-}$assignment; however, for the other states no definite assignment exists.

This paper reports on the study of $D_{J}$ spectroscopy in the $D^{*+} \pi^{-}$system through an amplitude analysis of the $B^{-} \rightarrow$ $D^{*+} \pi^{-} \pi^{-}$decay. ${ }^{2}$ The data sample corresponds to a total integrated luminosity of $4.7 \mathrm{fb}^{-1}$ of $p p$ collisions collected

\footnotetext{
${ }^{2}$ The inclusion of charge-conjugate processes is implied, unless stated.
} 
at center-of-mass energies of 7,8 and $13 \mathrm{TeV}$ with the $\mathrm{LHCb}$ detector. The 7 and $8 \mathrm{TeV}$ dataset is labeled in the following as "Run 1" data, and the $13 \mathrm{TeV}$ dataset as "Run 2" data.

The article is organized as follows. Section II gives details on the LHCb detector, while Sec. III is devoted to the description of the data selection procedure. Section IV describes the data features and Sec. V is devoted to the handling of the background and the efficiency model. In Sec. VI the amplitude analysis model is described, while Sec. VII and Sec. VIII give details on the fits to the data. The measurements of the partial branching fractions are given in Sec. IX and results are summarized in Sec. X.

\section{LHCb DETECTOR}

The $\mathrm{LHCb}$ detector $[15,16]$ is a single-arm forward spectrometer covering the pseudorapidity range $2<\eta<5$, designed for the study of particles containing $b$ or $c$ quarks. The detector includes a high-precision tracking system consisting of a silicon-strip vertex detector surrounding the $p p$ interaction region, a large-area silicon-strip detector located upstream of a dipole magnet with a bending power of about $4 \mathrm{Tm}$, and three stations of silicon-strip detectors and straw drift tubes placed downstream of the magnet. The polarity of the dipole magnet is reversed periodically throughout data-taking. The tracking system provides a measurement of the momentum of charged particles with relative uncertainty that varies from $0.5 \%$ at low momentum $(3 \mathrm{GeV})$ to $1.0 \%$ at $200 \mathrm{GeV}$. The minimum distance of a track to a primary vertex (PV) (defined as the location of a reconstructed $p p$ collision), the impact parameter (IP), is measured with a resolution of $\left(15+29 / p_{\mathrm{T}}\right) \mu \mathrm{m}$, where $p_{\mathrm{T}}$ is the component of the momentum transverse to the beam, in $\mathrm{GeV}$. Different types of charged hadrons are distinguished using information from two ring-imaging Cherenkov detectors. Photon, electron and hadron candidates are identified by a calorimeter system consisting of scintillating-pad and preshower detectors, an electromagnetic and a hadronic calorimeter. Muons are identified by a system composed of alternating layers of iron and multiwire proportional chambers.

The trigger consists of a hardware stage, based on information from the calorimeter and muon systems, followed by a software stage, in which all tracks with $p_{\mathrm{T}}>$ $500(300) \mathrm{MeV}$ are reconstructed for data collected at $\sqrt{s}=7 \mathrm{TeV}(8-13 \mathrm{TeV})$. The software trigger used in this analysis requires a two-, three- or four-track secondary vertex with significant displacement from the primary $p p$ interaction vertices. At least one charged particle must have $p_{\mathrm{T}}>1.7 \mathrm{GeV}$ and be inconsistent with originating from any PV. A multivariate algorithm [17] is used for the identification of secondary vertices consistent with the decay of a $b$ hadron.

In the offline selection, the objects that fired the trigger are associated with reconstructed particles. Selection requirements can therefore be made not only on the software trigger that fired, but also on whether the decision was due to the signal candidate, other particles produced in the $p p$ collision, or a combination of both. Both cases are retained for further analysis.

Simulated samples are used to characterize the detector response to signal and certain types of backgrounds. In the simulation, $p p$ collisions are generated using PYTHIA [18] with a specific LHCb configuration [19]. Decays of hadronic particles are described by EVTGEN [20], in which final state radiation is generated using Рнотоs [21]. The interaction of the generated particles with the detector and its response are implemented using the GEANT4 toolkit [22] as described in Ref. [23].

\section{SELECTION REQUIREMENTS}

The selection of the $B^{-}$meson is performed using the decay chain

$$
\begin{aligned}
p p & \rightarrow B^{-} X, & B^{-} \rightarrow D^{*+} \pi^{-} \pi^{-}, \\
D^{*+} & \rightarrow D^{0} \pi^{+}, & D^{0} \rightarrow K^{-} \pi^{+},
\end{aligned}
$$

where $X$ represents a system composed of any collection of charged or neutral particles. After applying selections on the quality of the $B^{-}$candidate tracks, further requirements are applied on their momenta, $p$, and transverse momenta, $p_{\mathrm{T}}$. The $D^{0}$ meson is reconstructed through its $K^{-} \pi^{+}$decay, applying loose particle identification criteria on both particles and good vertex quality requirements. The remaining tracks associated to the $B^{-}$final state form a $\pi^{+} \pi^{-} \pi^{-}$ system which defines the $B^{-}$decay vertex. Very loose particle-identification criteria are applied to the three pions together with good vertex-quality and impact-parameter constraints. The invariant mass of the above $\pi^{+} \pi^{-} \pi^{-}$ system is required to be below the physical boundary $m\left(\pi^{+} \pi^{-} \pi^{-}\right)<3.6 \mathrm{GeV}$. In the data collected at $\sqrt{s}=7$ and $8 \mathrm{TeV}(48.5 \%$ of the total dataset), the requirement is $m\left(\pi^{+} \pi^{-} \pi^{-}\right)<3.0 \mathrm{GeV}$, which also removes $1.2 \%$ of the signal. Although the loss in the Run 1 data is rather small, it produces a non-negligible distortion in the $B^{-}$Dalitz plot and in the $\pi^{-} \pi^{-}$invariant-mass distribution.

The momentum scale is calibrated using samples of $J / \psi \rightarrow \mu^{+} \mu^{-}$and $B^{+} \rightarrow J / \psi K^{+}$decays collected concurrently with the data sample used for this analysis [24,25]. The relative accuracy of this procedure is estimated to be $3 \times 10^{-4}$ using samples of other fully reconstructed $b$ hadrons, $\Upsilon$ and $K_{\mathrm{S}}^{0}$ mesons.

Figure 1(a) shows the $D^{0} \pi^{+}$mass spectrum, computed as $m\left(K^{-} \pi^{+} \pi_{\mathrm{s}}^{+}\right)-m\left(K^{-} \pi^{+}\right)+m_{D^{0}}^{\mathrm{PDG}}$ (here $\pi_{\mathrm{s}}^{+}$indicates the "slow pion" from the $D^{*+}$ decay and $m_{D^{0}}^{\mathrm{PDG}}$ indicates the known $D^{0}$ mass value), where a clean $D^{*+}$ signal can be observed.

The $D^{*+}$ candidate is selected within $3.5 \sigma$ of the fitted $D^{*+}$ mass value, where $\sigma=0.45 \mathrm{MeV}$ is the effective mass 

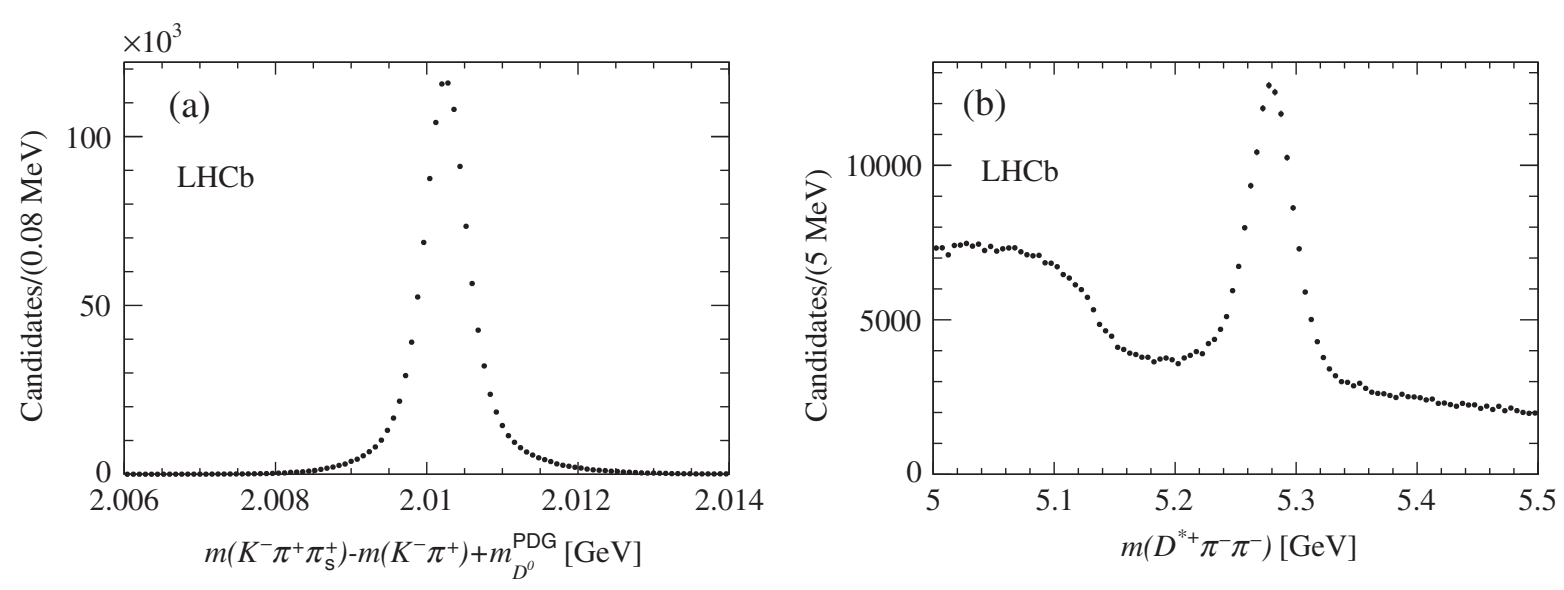

FIG. 1. Distribution of (a) $m\left(K^{-} \pi^{+} \pi_{\mathrm{s}}^{+}\right)-m\left(K^{-} \pi^{+}\right)+m_{D^{0}}^{\mathrm{PDG}}$ and (b) $D^{*+} \pi^{-} \pi^{-}$invariant masses for candidates after the selection on the $\chi^{2} /$ ndf from the fit to the $B^{-}$decay tree.

resolution obtained from a fit to the $D^{0} \pi^{+}$mass spectrum with a sum of two Gaussian functions. The $B^{-} \rightarrow D^{*+} \pi^{-} \pi^{-}$ decay is affected by background from $\bar{B}^{0} \rightarrow D^{*+} \pi^{-}$decays combined with a random $\pi^{-}$candidate in the event. This contribution populates the high-mass sideband of the $B^{-}$ signal and is removed if either $D^{*+} \pi^{-}$have a mass within $2 \sigma$ of the $\bar{B}^{0}$ known value [3], where $\sigma=18.5 \mathrm{MeV}$ is obtained from a Gaussian fit to the $\bar{B}^{0}$ mass distribution. The resulting $D^{*+} \pi^{-} \pi^{-}$mass distribution is shown in Fig 1(b), where the $B^{-}$signal can be observed over a significant background.

A significant source of background is due to $B^{-} \rightarrow$ $D^{*+} \pi^{-} \pi^{-} \pi^{0}$ or $\bar{B}^{0} \rightarrow D^{*+} \pi^{-} \pi^{-} \pi^{+}$decays, where a pion is not reconstructed. However the $B^{-} \rightarrow D^{*+} \pi^{-} \pi^{-}$mass combination from these final states populates the low-mass sideband of the $B^{-}$signal and does not extend into the signal region. A coherent source of background which could affect the signal region is due to $\bar{B}^{0} \rightarrow D^{*+} \pi^{-} \pi^{0}$ decays, where the $\pi^{0}$ meson is not reconstructed but is replaced by a random $\pi^{-}$candidate from the event. In this case the $D^{*+} \pi^{-}$system could have a definite spin-parity configuration; however, this contribution is found to be negligible. A possible source of background comes from the $B^{-} \rightarrow D^{*+} K^{-} \pi^{-}$decay, where the $K^{-}$candidate is misidentified as a pion. This background is kinematically confined in the lower sideband of the $B^{-}$signal. Its contribution relative to $B^{-} \rightarrow D^{*+} \pi^{-} \pi^{-}$ has been measured in Ref. [26] and found to be negligible. Therefore the background under the $B^{-}$signal is dominated by combinatorial background.

To reduce the combinatorial background while keeping enough signal for an amplitude analysis, a multivariate selection is employed, in the form of a likelihood ratio defined, for each event, as

$$
\mathcal{R}=\sum_{i=1}^{6} \log \left(P_{\mathrm{s}}(i) / P_{\mathrm{b}}(i)\right),
$$

where $i$ runs over a set of 6 variables and $P_{\mathrm{s}}(i)$ and $P_{\mathrm{b}}(i)$ are probability density functions (PDFs) of the signal and background contributions, respectively. The signal PDFs are obtained from simulated signal samples, while background PDFs are obtained from the $B^{-}$sideband regions, defined within 4.5-6.6 $\sigma$ on either side of the $B^{-}$mass peak, where $\sigma$ is obtained from the fit to the $D^{*+} \pi^{-} \pi^{-}$mass spectrum defined below. The variables used are: the $B^{-}$ decay length significance, defined as the ratio between the decay length and its uncertainty; the $B^{-}$transverse momentum; the $\chi^{2}$ of the primary vertex associated to the $B^{-}$meson; the $B^{-}$and $D^{*+}$ impact parameters with respect to the primary vertex; and the $\chi^{2} /$ ndf from the fit to the $B^{-} \rightarrow\left(D^{0}\left(\rightarrow K^{-} \pi^{+}\right) \pi_{\mathrm{s}}^{+}\right) \pi^{-} \pi^{-}$decay tree.

The choice of the selection value on the variable $\mathcal{R}$ is performed using an optimization procedure where the $D^{*+} \pi^{-} \pi^{-}$mass spectrum of candidates selected with

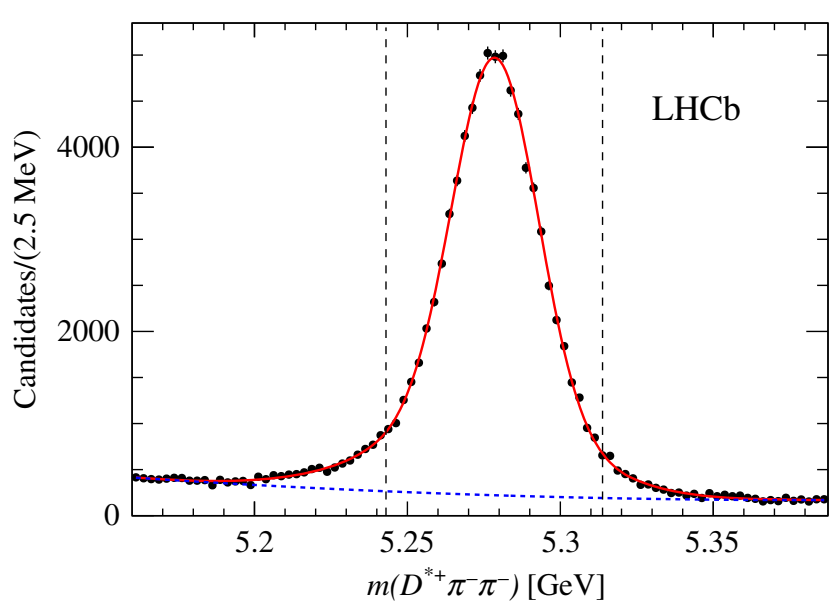

FIG. 2. Mass distribution for $D^{*+} \pi^{-} \pi^{-}$candidates after the selection $\mathcal{R}>0.5$. The full (red) line is the result from the fit while the dotted (blue) line describes the background. The vertical dashed lines indicate the signal region. 


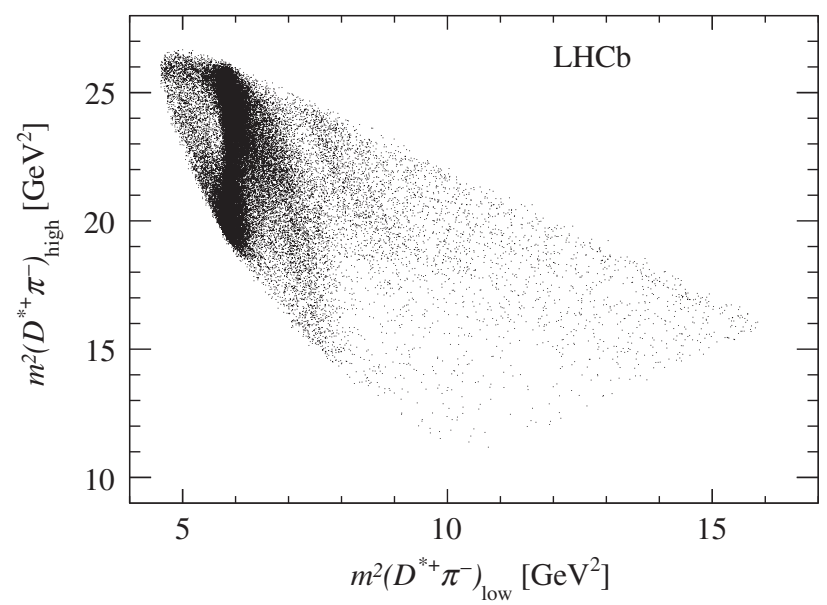

FIG. 3. Dalitz plot distribution for $B^{-} \rightarrow D^{*+} \pi^{-} \pi^{-}$candidates in Run 2 data.

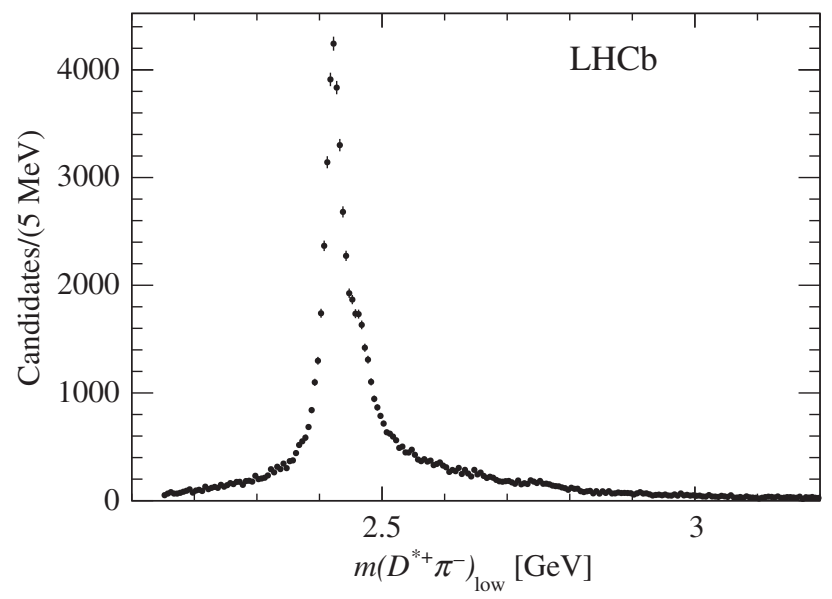

FIG. 4. Distribution of $m\left(D^{*+} \pi^{-}\right)_{\text {low }}$ for the total dataset.

increasing cut on $\mathcal{R}$ is fitted. The fits are performed using two Gaussian functions with a common mean to describe the $B^{-}$signal and a quadratic function for the background. Defining $\sigma$ as the weighted mean of the widths of the two Gaussian functions, the signal region is defined within $2 \sigma$, where $\sigma=17.7 \mathrm{MeV}$. For each selection the fit estimates the signal and background yields, $N_{\text {sig }}$ and $N_{\text {bkg }}$. From these quantities the purity $p=\frac{N_{\mathrm{sig}}}{N_{\mathrm{sig}}+N_{\mathrm{bkg}}}$ and the significance $s=\frac{N_{\text {sig }}}{\sqrt{N_{\text {sig }}+N_{\text {bkg }}}}$ are evaluated. To obtain both the largest purity and significance, the figure of merit $s \cdot p$ is evaluated. It has its maximum at $\mathcal{R}>0.5$ which is taken as the default selection. For this selection, Fig. 2 shows the resulting $D^{*+} \pi^{-} \pi^{-}$mass spectrum where the $B^{-}$signal is observed over small background.

For the above selection the signal purity is $p=0.9$, while the efficiency is $81.9 \%$. The yield within the signal region is 79120 , of which $48.5 \%$ and $51.5 \%$ are from Run 1 and Run 2, respectively. The purities of the two datasets are found to be the same. The number of events with multiple $B^{-}$candidate combinations is negligible.

\section{THE $B^{-} \rightarrow D^{*+} \pi^{-} \pi^{-}$DALITZ PLOT}

The $B^{-} \rightarrow D^{*+} \pi^{-} \pi^{-}$decay mode contains two indistinguishable $\pi^{-}$mesons, giving two $D^{*+} \pi^{-}$mass combinations. In the following, $m\left(D^{*+} \pi^{-}\right)_{\text {low }}$ and $m\left(D^{*+} \pi^{-}\right)_{\text {high }}$ indicate the lower and higher values of the two mass combinations, respectively. The $B^{-}$Dalitz plot, described as a function of $m^{2}\left(D^{*+} \pi^{-}\right)_{\text {high }}$ and $m^{2}\left(D^{*+} \pi^{-}\right)_{\text {low }}$, is shown in Fig. 3 for Run 2 data.

The Dalitz plot contains clear vertical bands in the $6 \mathrm{GeV}^{2}$ mass region, due to the presence of the wellknown $D_{1}(2420)$ and $D_{2}^{*}(2460)$ resonances. The presence of further weaker bands can be observed in the higher mass region. The prominent presence of the above two resonances can be observed in the $m\left(D^{*+} \pi^{-}\right)_{\text {low }}$ projection, shown in Fig. 4 for the total dataset. On the other hand, the presence of additional states is rather weak in the mass projection and therefore an angular analysis is needed to separate the different contributions.

The following angles are useful in discriminating between different $J^{P}$ contributions:

$\theta_{H}$, the helicity angle defined as the angle between the $\pi_{\mathrm{s}}^{+}$direction in the $D^{0} \pi_{\mathrm{s}}^{+}$rest frame and the $D^{0} \pi_{\mathrm{s}}^{+}$ direction in the $D^{0} \pi_{\mathrm{s}}^{+} \pi^{-}$rest frame [see Fig. 5(a)];

$\theta$, the helicity angle defined as the angle formed by the $\pi_{1}^{-}$direction in the $D^{*+} \pi_{1}^{-}$rest frame and the $D^{*+} \pi_{1}^{-}$ direction in the $D^{*+} \pi_{1}^{-} \pi_{2}^{-}$rest frame [see Fig. 5(b)];

$\gamma$, the angle in the $D^{*+} \pi^{-} \pi^{-}$rest frame formed by the $\pi_{\mathrm{s}}^{+}$ direction in the $D^{0} \pi_{\mathrm{s}}^{+}$rest frame and the normal to the $D^{*+} \pi^{-} \pi^{-}$plane [see Fig. 5(c)].

The angle $\theta_{H}$ is useful to discriminate between natural and unnatural spin-parity resonances for which the expected angular distributions are $\sin ^{2} \theta_{H}$ and $1-h \cos ^{2} \theta_{H}$ (a)

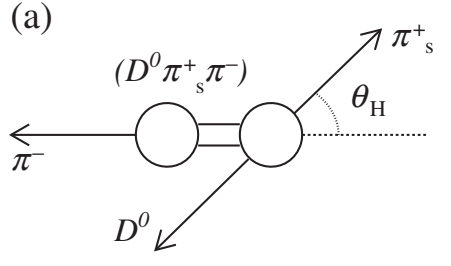

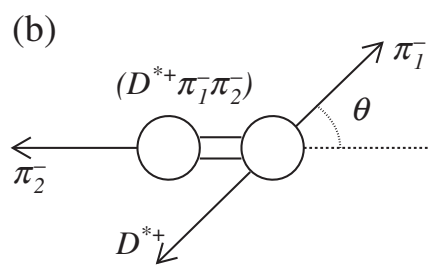

FIG. 5. Definition of the angles (a) $\theta_{H}$, (b) $\theta$ and (c) $\gamma$. 


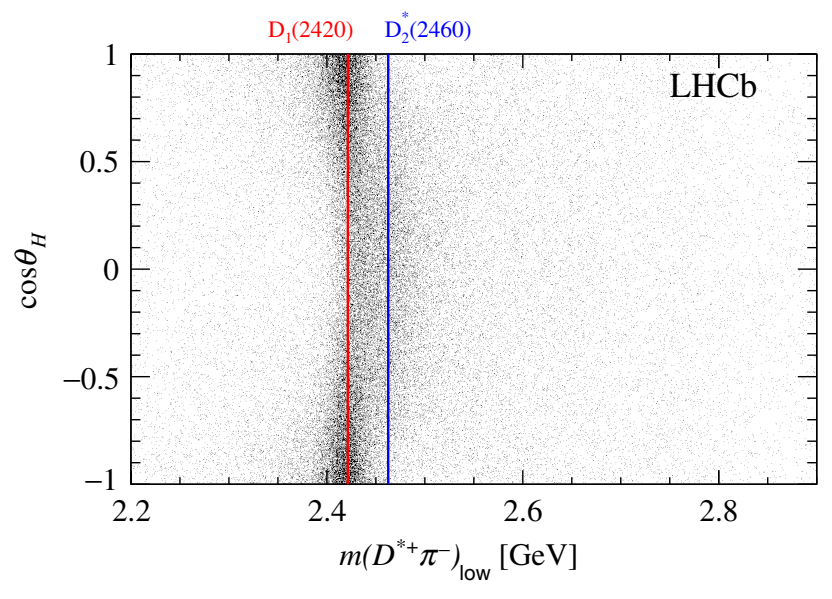

FIG. 6. Two-dimensional distribution of $\cos \theta_{H} \operatorname{vs} m\left(D^{*+} \pi^{-}\right)_{\text {low }}$. The vertical lines indicate the positions of the $D_{1}(2420)$ and $D_{2}^{*}(2460)$ resonances.

(where $h<1$ depends on the properties of the decay), respectively, except for $J^{P}=0^{-}$where a $\cos ^{2} \theta_{H}$ distribution is expected. Figure 6 shows the two-dimensional distribution of $\cos \theta_{H}$ vs $m\left(D^{*+} \pi^{-}\right)_{\text {low }}$. The two vertical bands are due to the $D_{1}(2420)$ and $D_{2}^{*}(2460)$ states which exhibit the expected $1-h \cos ^{2} \theta_{H}$ and $\sin ^{2} \theta_{H}$ distributions, respectively.
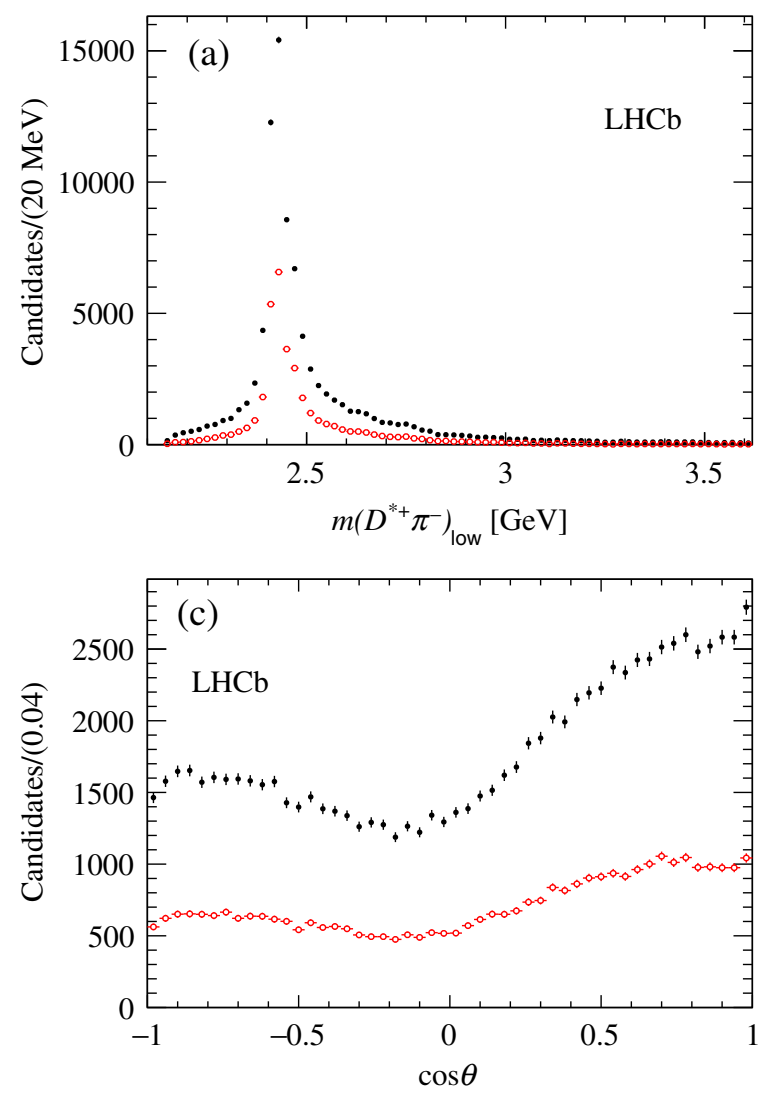

\section{BACKGROUND AND EFFICIENCY}

\section{A. Background model}

The background model is obtained from the data in the signal region using the method of signal subtraction. Using the $\mathcal{R}$ variable defined in Eq. (2), two datasets are extracted, (a) with low purity $\left(\mathcal{R}>0.0, p_{a}=0.865\right.$ and signal yield $\left.N_{a}=77644\right)$, (b) with high purity $\left(\mathcal{R}>2.5, p_{b}=0.949\right.$ and signal yield $N_{b}=34019$ ). The background distribution for a given variable is then obtained by subtracting the highpurity distributions, scaled by the factor $N_{a} / N_{b}$, from the low-purity distributions. The variables $m\left(D^{*+} \pi^{-}\right)_{\text {low }}$ and $\cos \theta$ are found to be independent and different for signal and background, therefore the resulting background model is obtained by the product of the PDFs of these distributions. Figure 7(a) shows the $m\left(D^{*+} \pi^{-}\right)_{\text {low }}$ distribution for the lowpurity (filled points) and high-purity (open points) selections. Figure 7(b) shows the signal-subtracted distribution, where no significant resonant structure is seen. The superimposed curve is the result from a fit performed using the sum of two exponential functions multiplied by the $B^{-}$phasespace factor. Similarly, Fig. 7(c) shows the $\cos \theta$ distribution for the low-purity and high-purity samples, and Fig. 7(d) shows the signal-subtracted distribution. The curve is the result of a fit using a 6th order polynomial function.
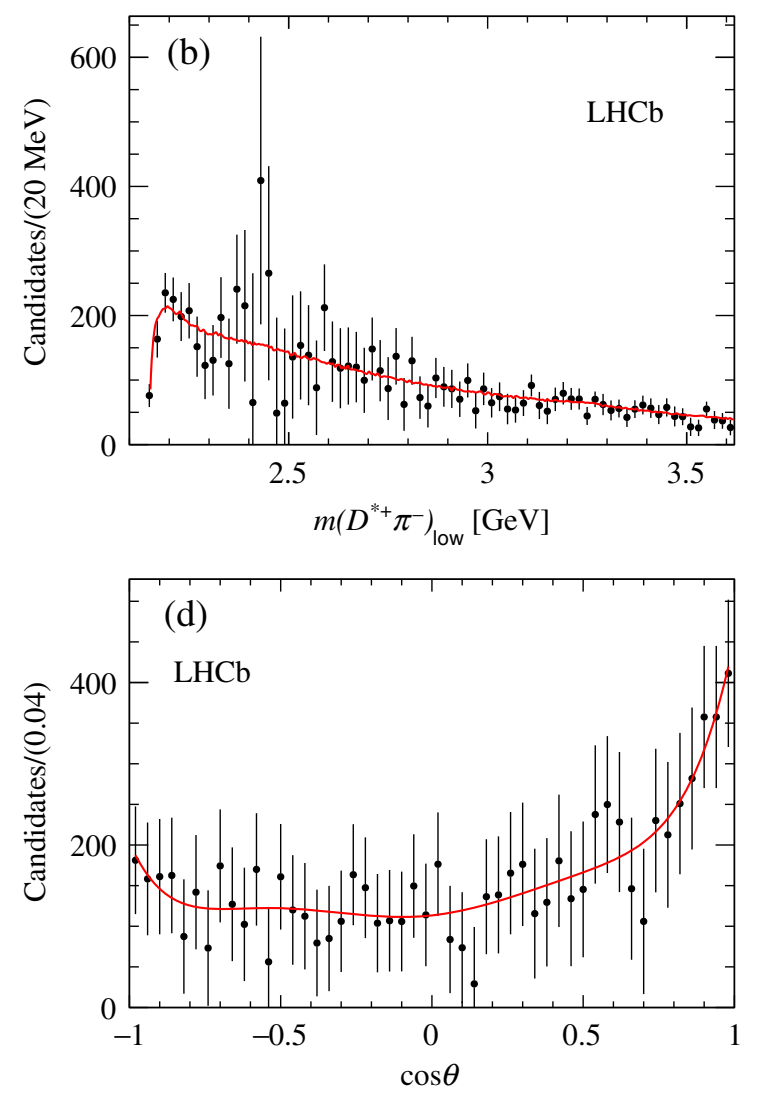

FIG. 7. Distribution for low/high-purity selection (filled black/open red points) for (a) $m\left(D^{*+} \pi^{-}\right.$) low and (c) $\cos \theta$. Signal-subtracted distributions for (b) $m\left(D^{*+} \pi^{-}\right)_{\text {low }}$ and (d) $\cos \theta$ with superimposed fit curves described in the text. 


\section{B. Efficiency}

The efficiency is computed using simulated samples of signal decays analyzed using the same procedure as for data. Due to the different trigger and reconstruction methods, the efficiency is computed separately for Run 1 and Run 2 data. It is found that the efficiency mainly depends on the variables, $m\left(D^{*+} \pi^{-}\right)$and $\cos \theta$. Weak or no dependence is found on other variables. The efficiency model is obtained by dividing the simulated sample into 22 slices in $\log \left(m\left(D^{*+} \pi^{-}\right) / \mathrm{MeV}\right)$ and fitting the $\cos \theta$ distribution for each slice using 5th order polynomial functions. The efficiency for a given value of $\log \left(m\left(D^{*+} \pi^{-}\right) / \mathrm{MeV}\right)$ is then obtained by linear interpolation between two adjacent bins.

Figure 8 shows the interpolated efficiency maps in the $\left(\log \left(m\left(D^{*+} \pi^{-}\right) / \mathrm{MeV}\right), \cos \theta\right)$ plane, separately for Run 1 and Run 2 . The empty region in Run 1 data is caused by the requirement $m\left(\pi^{+} \pi^{-} \pi^{-}\right)<3.0 \mathrm{GeV}$. Although this region is populated by a small fraction of signal, estimated using Run 2 data, this introduces some uncertainty in the description of the Run 1 data.

The mass resolution is studied as function of $m\left(D^{*+} \pi^{-}\right)$ using simulation. For each slice in $m\left(D^{*+} \pi^{-}\right)$the difference between the generated and reconstructed mass is computed and the resulting distributions are fitted using the sum of two Gaussian functions. The effective resolution $\sigma$, increases almost linearly from $\sigma=4 \mathrm{MeV}$ at $2.4 \mathrm{GeV}$ to $\sigma=7 \mathrm{MeV}$ at $3.0 \mathrm{GeV}$. This value of the mass resolution is much smaller than the minimum width of the known resonances present in the $D^{*+} \pi^{-}$mass spectrum (for $\left.D_{1}(2420), \Gamma=31 \mathrm{MeV}\right)$; therefore, in the following, resolution effects are ignored.

\section{AMPLITUDE ANALYSIS}

An amplitude analysis of the four-body decay

$$
B^{-} \rightarrow\left(D^{0} \pi^{+}\right)_{D^{*+}} \pi_{1}^{-} \pi_{2}^{-},
$$

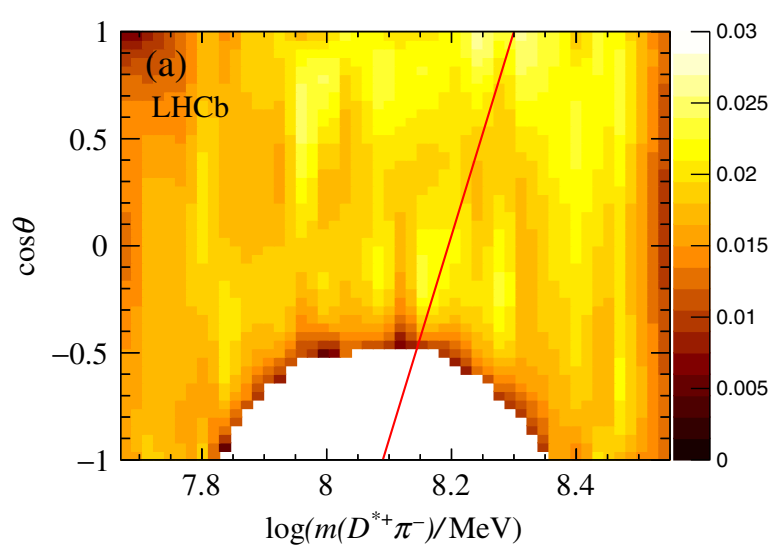

where $\pi_{1}^{-}$and $\pi_{2}$ indicate the two indistinguishable pions, is performed to extract the fractions and the phases of the charmed resonances contributing to the decay and to measure their parameters and quantum numbers. All the amplitudes are symmetrized according to the exchange of the $\pi_{1}^{-}$with $\pi_{2}^{-}$mesons.

\section{A. Description of the amplitudes}

The amplitudes contributing to the decay are parametrized using the nonrelativistic Zemach tensors formalism [27-29]. It is assumed that reaction (3) proceeds as

$$
B^{-} \rightarrow R^{0} \pi_{2}^{-},
$$

where $R^{0}$ is an intermediate charmed meson resonance which decays as

$$
R^{0} \rightarrow D^{*+}\left(\rightarrow D^{0} \pi_{\mathrm{s}}^{+}\right) \pi_{1}^{-} .
$$

Reaction (4) is a weak decay and does not conserve parity while reaction (5) is a strong decay and conserves both angular momentum and parity. The four particles in the final state are labeled as

$$
D^{0}(1), \quad \pi_{\mathrm{s}}^{+}(2), \quad \pi_{1}^{-}(3), \quad \pi_{2}^{-}(4) .
$$

In the description of the decay $R^{0} \rightarrow D^{0} \pi_{\mathrm{s}}^{+} \pi_{1}^{-}$, the 3 -vectors $\boldsymbol{p}_{\boldsymbol{i}}(i=1,2,3)$ indicate the momenta of the particles in the $D^{0} \pi_{\mathrm{s}}^{+} \pi_{1}^{-}$rest frame and $L$ indicates the angular momentum between the $D^{0} \pi_{\mathrm{s}}^{+}$system and the $\pi_{1}^{-}$meson. For the resonance $D^{*+}$ decaying as $D^{*+} \rightarrow D^{0} \pi_{\mathrm{s}}^{+}$, the decay products $D^{0}$ and $\pi_{\mathrm{s}}^{+}$having 3-momenta $\boldsymbol{p}_{1}, \boldsymbol{p}_{2}$ and masses $m_{1}$ and $m_{2}$, the 3 -vector $\boldsymbol{t}_{\mathbf{3}}$ is defined as

$$
\boldsymbol{t}_{\mathbf{3}}=\boldsymbol{p}_{\mathbf{1}}-\boldsymbol{p}_{\mathbf{2}}-\left(\boldsymbol{p}_{\mathbf{1}}+\boldsymbol{p}_{\mathbf{2}}\right) \frac{m_{1}^{2}-m_{2}^{2}}{m_{12}^{2}},
$$

with $m_{12}$ indicating the $D^{0} \pi_{\mathrm{s}}^{+}$invariant mass. To describe the decay $B^{-} \rightarrow R^{0} \pi_{2}^{-}$, the 3-vector $\boldsymbol{q}_{\mathbf{4}}$ indicates the momentum of $\pi_{2}^{-}$in the $B^{-}$rest frame.

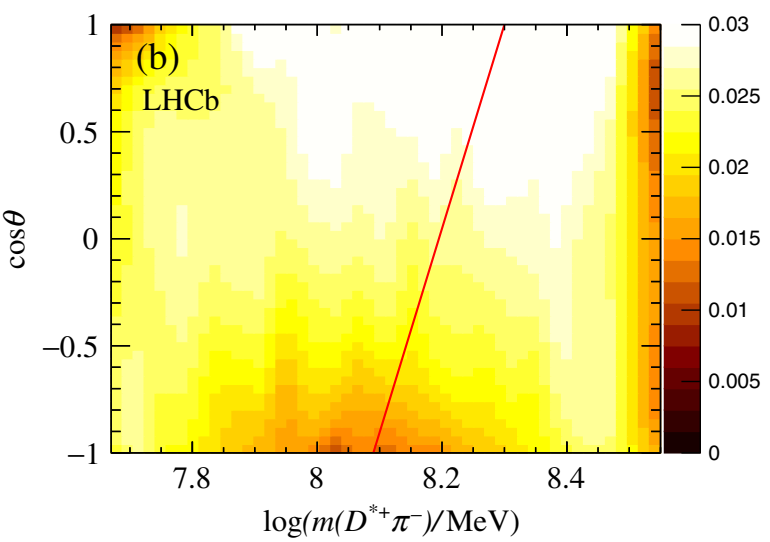

FIG. 8. Interpolated efficiency as a function of $\log \left(m\left(D^{*+} \pi^{-}\right) / \mathrm{MeV}\right)$ and $\cos \theta$ for (a) Run 1 and (b) Run 2 data. The line indicates the regions where $\log \left(m\left(D^{*+} \pi^{-}\right) / \mathrm{MeV}\right)$ is taken as $\log \left(m\left(D^{*+} \pi^{-}\right)_{\operatorname{low}} / \mathrm{MeV}\right)$ (left side of the line) and as $\log \left(m\left(D^{*+} \pi^{-}\right)_{\text {high }} / \mathrm{MeV}\right)($ right side of the line). 
The amplitudes are obtained as follows:

(i) a symmetric and traceless tensor of rank $L, \boldsymbol{P}$, constructed with $\boldsymbol{p}_{\mathbf{3}}$ is used to describe the orbital angular momentum $L$;

(ii) a symmetric and traceless tensor of rank $S, \boldsymbol{T}$, constructed with $\boldsymbol{t}_{\mathbf{3}}$ is used to describe the spin of intermediate resonances;

(iii) the tensors $\boldsymbol{P}$ and $\boldsymbol{T}$ are then combined into a tensor $\boldsymbol{J}$ of rank $J$ to obtain the total spin $J$ of the $D^{0} \pi^{+} \pi_{1}^{-}$ system;

(iv) a symmetric and traceless tensor, $Q$, of rank $J$ constructed with $\boldsymbol{q}_{4}$ is used to describe the orbital angular momentum between $R^{0}$ and $\pi_{2}^{-}$;

(v) the scalar product of the two tensors $\boldsymbol{J}$ and $\boldsymbol{Q}$ gives the scalar which represents the 0 spin of the $B^{-}$meson.

The resonance $R^{0}$, having a given $J^{P}$, decays into a $1^{-}$resonance $\left(D^{*+}\right)$ and a $0^{-}$particle with a given orbital angular momentum $L$. In a first approach, the resonance line shape is described by a complex relativistic Breit-Wigner function, $\mathrm{BW}\left(m_{123}\right)$, with appropriate Blatt-Weisskopf centrifugal barrier factors [14,30] which are computed assuming a radius $4.5 \mathrm{GeV}^{-1}$. In a second approach the resonance line shapes are described by the quasi-modelindependent method (QMI) [31,32], described later.

The list of the amplitudes used in the present analysis is given in Table I. The $J^{P}=0^{-}$nonresonant contribution term is omitted because it is found to be negligible.

\section{B. Amplitude analysis fit}

The amplitude analysis of the $D^{*+} \pi^{-} \pi^{-}$candidates in the $B^{-}$mass region is performed using unbinned maximumlikelihood fits. The likelihood function is written as

$\mathcal{L}=\prod_{n=1}^{N}\left[p \cdot \epsilon\left(\boldsymbol{x}_{\boldsymbol{n}}\right) \frac{\sum_{i, j} c_{i} c_{j}^{*} A_{i}\left(\boldsymbol{x}_{\boldsymbol{n}}\right) A_{j}^{*}\left(\boldsymbol{x}_{\boldsymbol{n}}\right)}{\sum_{i, j} c_{i} c_{j}^{*} I_{A_{i} A_{j}^{*}}}+(1-p) B\left(\boldsymbol{z}_{\boldsymbol{n}}\right)\right]$,

where

(i) $N$ is the number of candidates in the signal region; (ii) for the $n$th event, $\boldsymbol{x}_{\boldsymbol{n}}$ is the set of variables describing the 4-body $B$ meson decay;

(iii) $\epsilon\left(\boldsymbol{x}_{\boldsymbol{n}}\right)$ is the efficiency function;

(iv) $A_{i}\left(\boldsymbol{x}_{\boldsymbol{n}}\right)$ represents the $i$ th complex signal-amplitude contribution;

(v) $c_{i}$ is the complex intensity of the $i$ th signal component; the $c_{i}$ parameters are allowed to vary during the fit process;

(vi) $I_{A_{i} A_{j}^{*}}=\int A_{i}(\boldsymbol{x}) A_{j}^{*}(\boldsymbol{x}) \epsilon(\boldsymbol{x}) \mathrm{d} \boldsymbol{x}$ are normalization integrals; numerical integration is performed on phasespace-generated decays with the $B^{-}$signal line shape generated according to the experimental distribution;

(vii) $p$ is the signal purity obtained from the fit to the $D^{*+} \pi^{-} \pi^{-}$mass spectrum;

(viii) $B\left(z_{n}\right)$ is the normalized background contribution, parametrized as a function of the two variables described in Sec. V B.

The efficiency-corrected fraction $f_{i}$ due to a resonant or nonresonant contribution $i$ is defined as follows:

$$
f_{i} \equiv \frac{\left|c_{i}\right|^{2} \int\left|A_{i}(\boldsymbol{x})\right|^{2} \mathrm{~d} \boldsymbol{x}}{\sum_{i, j} c_{i} c_{j}^{*} I_{A_{i} A_{j}^{*}}} .
$$

The $f_{i}$ fractions do not necessarily sum to 1 because of interference effects. The uncertainty for each $f_{i}$ fraction is evaluated by propagating the full covariance matrix obtained from the fit. Similarly, the efficiency-corrected interference fractional contribution $f_{i j}$, for $i<j$ is defined as

$$
f_{i j} \equiv \frac{\int 2 \mathcal{R} e\left[c_{i} c_{j}^{*} A_{i}(\boldsymbol{x}) A_{j}^{*}(\boldsymbol{x})\right] \mathrm{d} \boldsymbol{x}}{\sum_{i, j} c_{i} c_{j}^{*} I_{A_{i} A_{j}^{*}}} .
$$

The amplitude analysis is started by including, one by one, all the possible charmed resonance contributions with masses and widths listed in Ref. [3]. Resonances are kept if a significant likelihood increase $(\Delta(2 \log \mathcal{L})>3)$ is observed. The list of the states giving significant contributions at the end of the process is given in the upper

TABLE I. List of the amplitudes used in the present analysis.

\begin{tabular}{llc}
\hline \hline$J^{P}$ & $L$ & Amplitude \\
\hline $0^{-}$ & 1 & $\mathrm{BW}\left(m_{123}\right)\left[\boldsymbol{t}_{\mathbf{3}} \cdot \boldsymbol{p}_{\mathbf{3}}\right]$ \\
$1^{+} S$ & 0 & $\mathrm{BW}\left(m_{123}\right)\left[\boldsymbol{t}_{\mathbf{3}} \cdot \boldsymbol{q}_{\mathbf{4}}\right]$ \\
$1^{+} D$ & 2 & $\mathrm{BW}\left(m_{122}\right)\left[\boldsymbol{p}_{\mathbf{3}}\left(\boldsymbol{t}_{\mathbf{3}} \cdot \boldsymbol{p}_{\mathbf{3}}\right)-\frac{1}{3}\left(\boldsymbol{p}_{\mathbf{3}} \cdot \boldsymbol{p}_{\mathbf{3}}\right) \boldsymbol{t}_{\mathbf{3}}\right] \cdot \boldsymbol{q}_{\mathbf{4}}$ \\
$1^{-}$ & 1 & $\mathrm{BW}\left(m_{123}\right)\left[\left(\boldsymbol{t}_{\mathbf{3}} \times \boldsymbol{p}_{\mathbf{3}}\right) \cdot \boldsymbol{q}_{\mathbf{4}}\right]$ \\
$2^{-} P$ & 1 & $\mathrm{BW}\left(m_{123}\right)\left[\frac{1}{2}\left(t_{3}^{i} p_{3}^{j}+t_{3}^{j} p_{3}^{i}\right)-\frac{1}{3}\left(\boldsymbol{t}_{\mathbf{3}} \cdot \boldsymbol{p}_{\mathbf{3}}\right) \delta^{i j}\right] \cdot\left[q_{4}^{i} q_{4}^{j}-\frac{1}{3}\left|q_{4}\right|^{2} \delta^{i j}\right]$ \\
$2^{-} F$ & 3 & $\mathrm{BW}\left(m_{123}\right)\left[\left(\boldsymbol{t}_{\mathbf{3}} \cdot \boldsymbol{p}_{\mathbf{3}}\right)\left(p_{3}^{i} p_{3}^{j}-\frac{1}{3}\left|p_{3}\right|^{2} \delta^{i j}\right)\right] \cdot\left[q_{4}^{i} q_{4}^{j}-\frac{1}{3}\left|q_{4}\right|^{2} \delta^{i j}\right]$ \\
$2^{+}$ & 2 & $\mathrm{BW}\left(m_{123}\right)\left[\frac{1}{2}\left[\left(\boldsymbol{t}_{\mathbf{3}} \times \boldsymbol{p}_{\mathbf{3}}\right)^{i} p_{3}^{j}+p_{3}^{i}\left(\boldsymbol{t}_{\mathbf{3}} \times \boldsymbol{p}_{\mathbf{3}}\right)^{j}\right]-\frac{1}{3}\left[\left(\boldsymbol{t}_{\mathbf{3}} \times \boldsymbol{p}_{\mathbf{3}}\right) \cdot \boldsymbol{p}_{\mathbf{3}}\right] \delta^{i j} \cdot\left[q_{4}^{i} q_{4}^{j}-\frac{1}{3}\left|q_{4}\right|^{2} \delta^{i j}\right]\right.$ \\
$3^{-}$ & 3 & $\mathrm{BW}\left(m_{123}\right)\left[\left(\boldsymbol{t}_{\mathbf{3}} \times \boldsymbol{p}_{\mathbf{3}}\right)^{i} p_{3}^{j} p_{3}^{k}+\left(\boldsymbol{t}_{\mathbf{3}} \times \boldsymbol{p}_{\mathbf{3}}\right)^{k} p_{3}^{i} p_{3}^{j}+\left(\boldsymbol{t}_{\mathbf{3}} \times \boldsymbol{p}_{\mathbf{3}}\right)^{j} p_{3}^{i} p_{3}^{k}\right)$ \\
& & $\left.-\frac{1}{5}\left[\delta^{i j}\left(\boldsymbol{t}_{\mathbf{3}} \times \boldsymbol{p}_{\mathbf{3}}\right)^{k}+\delta^{i k}\left(\boldsymbol{t}_{\mathbf{3}} \times \boldsymbol{p}_{\mathbf{3}}\right)^{j}+\delta^{j k}\left(\boldsymbol{t}_{\mathbf{3}} \times \boldsymbol{p}_{\mathbf{3}}\right)^{i}\right]\left|p_{3}\right|^{2}\right] \cdot \frac{1}{5}\left[\left(p_{4}^{i} p_{4}^{j} p_{4}^{k}+p_{4}^{k} p_{4}^{i} p_{4}^{j}+p_{4}^{j} p_{4}^{p_{4}} p_{4}^{k}\right)-p_{4}^{2}\left(\delta^{i j} p_{4}^{k}+\delta^{i k} p_{4}^{j}+\delta^{j k} p_{4}^{i}\right)\right]$ \\
\hline \hline
\end{tabular}


TABLE II. Resonance parameters from the amplitude analysis. The first uncertainty is statistical, the second systematic. The upper part reports the resonance parameters obtained from the amplitude analysis described in Sec. VII, the lower part those obtained from the mixing analysis described in Sec. VIII C. The labels indicating the spin-parity of $D_{0}(2550), D_{1}^{*}(2600)$, and $D_{2}(2740)$ resonances are updated, with respect to those reported in Ref. [3], according to the results from the amplitude analysis reported in this work. The $D_{2}^{*}(2460)$ parameters are fixed to the world averages.

\begin{tabular}{llccc}
\hline \hline Resonance & $J^{P}$ & Mass $[\mathrm{MeV}]$ & Width $[\mathrm{MeV}]$ & Significance $(\sigma)$ \\
\hline$D_{1}(2420)$ & $1^{+}$ & $2424.8 \pm 0.1 \pm 0.7$ & $33.6 \pm 0.3 \pm 2.7$ & $309 \pm 9 \pm 28$ \\
$D_{1}(2430)$ & $1^{+}$ & $2411 \pm 3 \pm 9$ & $47.5 \pm 1.1$ & $199 \pm 5 \pm 17$ \\
$D_{2}^{*}(2460)$ & $2^{+}$ & $2460.56 \pm 0.35$ & $149 \pm 4 \pm 20$ & $102 \pm 6 \pm 26$ \\
$D_{0}(2550)$ & $0^{-}$ & $2518 \pm 2 \pm 7$ & $66 \pm 10 \pm 14$ & 60 \\
$D_{1}^{*}(2600)$ & $1^{-}$ & $2641.9 \pm 1.8 \pm 4.5$ & $31.5 \pm 0.1 \pm 2.1$ & 8.7 \\
$D_{2}(2740)$ & $2^{-}$ & $2751 \pm 3 \pm 7$ & $444 \pm 11 \pm 36$ \\
$D_{3}^{*}(2750)$ & $3^{-}$ & $2753 \pm 4 \pm 6$ & $2423.7 \pm 0.1 \pm 0.8$ & \\
$D_{1}$ & $1^{+}$ & $2452 \pm 4 \pm 15$ & $1^{+}$ & \\
$D_{1}^{\prime}$ & & &
\end{tabular}

section of Table II. The fit procedure is tested on pseudoexperiments using different combinations of amplitudes, input fractions and phases, obtaining a good agreement between generated and fitted values.

The quality of the description of the data is tested by the $\chi^{2} /$ ndf, defined as the sum of two $\chi^{2}$ values, calculated from the two two-dimensional distributions $\left(m^{\prime}, \cos \theta_{H}\right)$ and $(\cos \theta, \cos \gamma)$ as

$$
\chi^{2}=\sum_{i}\left(N_{i}^{\text {model }}-N_{i}^{\text {data }}\right)^{2} / N_{i}^{\text {model }} .
$$

Here $N_{i}^{\text {model }}$ and $N_{i}^{\text {data }}$ are the fit predictions and observed yields in each bin of the two-dimensional distributions. The variable ndf is defined as ndf $=N_{\text {cells }}-N_{\text {par }}$, where $N_{\text {cells }}$ is the number of bins having at least 6 entries and $N_{\text {par }}$ is the number of free parameters in the fit. The variable $m^{\prime}$, defined in the range $0-1$, is computed as

$$
m^{\prime}=\frac{1}{\pi} \arccos \left(2 \frac{m\left(\pi^{-} \pi^{-}\right)-m_{\pi^{-} \pi^{-}}^{\min }}{m_{\pi^{-} \pi^{-}}^{\max }-m_{\pi^{-} \pi^{-}}^{\min }}-1\right),
$$

where $m_{\pi^{-} \pi^{-}}^{\max }=m_{B^{-}}-m_{D^{*+}}$ and $m_{\pi^{-} \pi^{-}}^{\min }=2 m_{\pi^{-}}$.

\section{FITS TO THE DATA USING QUASI-MODEL- INDEPENDENT AMPLITUDES}

It is found in several analyses that the mass terms of some amplitudes may not be well described by Breit-Wigner functions, because they are broad or because additional contributions may be present at higher mass. Therefore, for a given value of $J^{P}$, a quasi-model-independent method is tested to describe the amplitude, while leaving all the other resonances described by Breit-Wigner functions. The method is also used to perform a scan of the mass spectrum to search for additional resonances.

The $D^{*+} \pi^{-}$mass spectrum is divided into 31 slices with nonuniform bin widths and, for a given contributing resonance, the complex Breit-Wigner term is replaced by a set of 31 complex coefficients (magnitude and phase) which are free to float. These values are fixed to arbitrary values in one bin, at a mass value in the $2.42-2.60 \mathrm{GeV}$ range, depending on the amplitude and therefore the set of additional free parameters is reduced to 60 .

The largest amplitude, usually the $1^{+} D$ amplitude, is taken as the reference wave. Due to the large number of fit parameters, QMI amplitudes can only be introduced one by one. The fit is performed using as free parameters the real and imaginary parts of the amplitude in each bin of the $D^{*+} \pi^{-}$mass spectrum. The search for the QMI parameters is performed using a random search, starting from zero in each mass bin. The fitted solution is then given as input to a second iteration modifying the value for the fixed bin to the average value obtained from the two adjacent bins. Obvious spikes are smoothed in the input of the second iteration. Normally the second iteration converges and is able to compute the full covariance matrix. The fitted QMI amplitude is then modeled through a cubic-spline interpolation function.

The method is tested using different initial values for the first iteration. In all cases the fit converges to the same solution. It is also tested with simulation obtaining good agreement between input and fitted values of the amplitudes.

The process starts with a QMI fit to the $J^{P}=1^{+} S$ amplitude, including all the amplitudes listed in the upper part of Table II and described by Breit-Wigner functions with initial parameter values fixed to those reported in Ref. [3]. In this fit, due to significant interference effects between the $1^{+} D$, the narrow $1^{+} S$ and the broad $1^{+} S$ amplitudes, the narrow $1^{+} D / 1^{+} S D_{1}(2420)$ parameters, described by a Breit-Wigner function, are left free as well. The resulting parameters for $D_{1}(2420)$ resonance are given in Table II. Statistical significances are computed as the fitted fraction divided by its statistical uncertainty.

The QMI $J^{P}=1^{+} S$ amplitude is then fixed and a QMI analysis of the $J^{P}=0^{-}$is performed. The process continues by fixing the $J^{P}=0^{-}$QMI amplitude and leaving, 

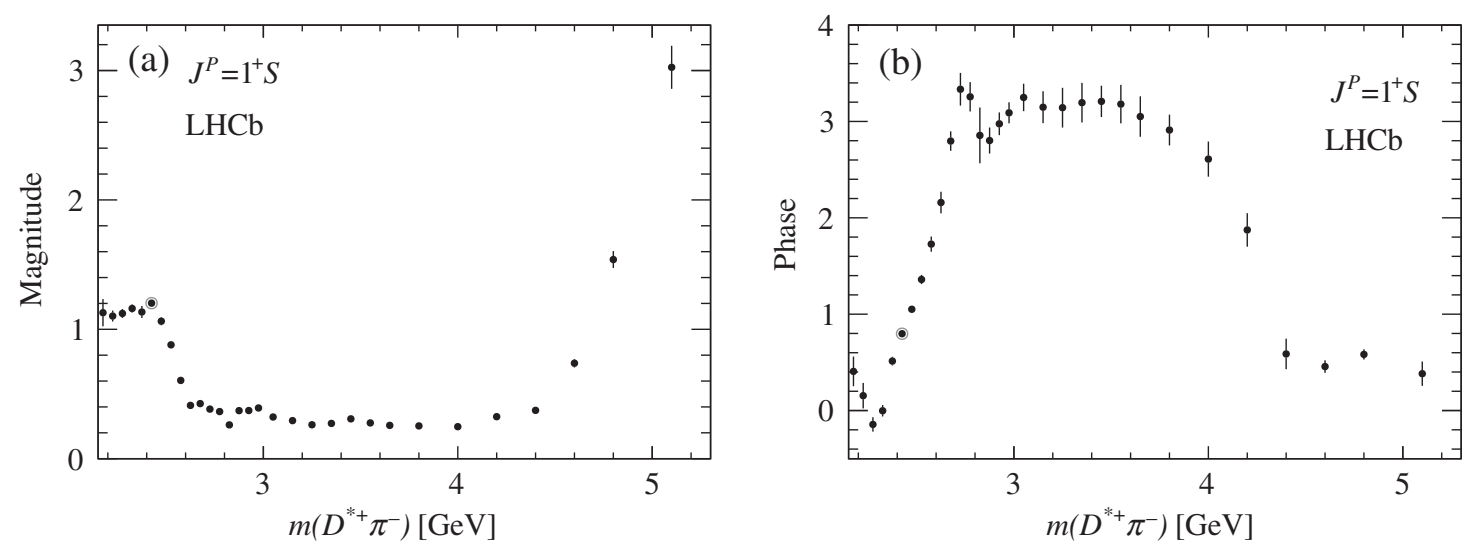

FIG. 9. (a) Magnitude and (b) phase of the $1^{+} S$ amplitude from the QMI method. The red circles indicate the mass bin where the complex amplitude has been fixed.

one by one, free Breit-Wigner parameters for all the resonances listed in the upper part of Table II. The parameters of the $D_{2}^{*}(2460)$ resonances are fixed to the world averages because they are well determined. The process is iterative, with QMI analyses of the $J^{P}=$ $1^{+} S$ and $J^{P}=0^{-}$amplitudes and free parameters for the resonances described by Breit-Wigner functions repeated several times, until the process converges and no significant variation of the free parameters is observed. The resulting fitted parameters of $D_{1}^{*}(2600), D_{2}(2740)$, and $D_{3}^{*}(2750)$ amplitudes are listed in Table II. To obtain the parameters of the broad $D_{1}(2430)$ resonance, a fit is performed with the QMI model for the $J^{P}=1^{+} S$ amplitude replaced by the Breit-Wigner function model. Similarly, to obtain the $D_{0}(2550)$ parameters, the QMI model for the $J^{P}=0^{-}$ amplitude is replaced by the Breit-Wigner function. The presence of a broad $J^{P}=1^{+} D D_{1}(2430)$ contribution has been tested but excluded from the final fit. Its effect, due to the presence the broad $J^{P}=1^{+} S$ resonance, is to produce large interference effects so that the total fraction increases to large and rather unphysical values without significantly improving the fit quality.

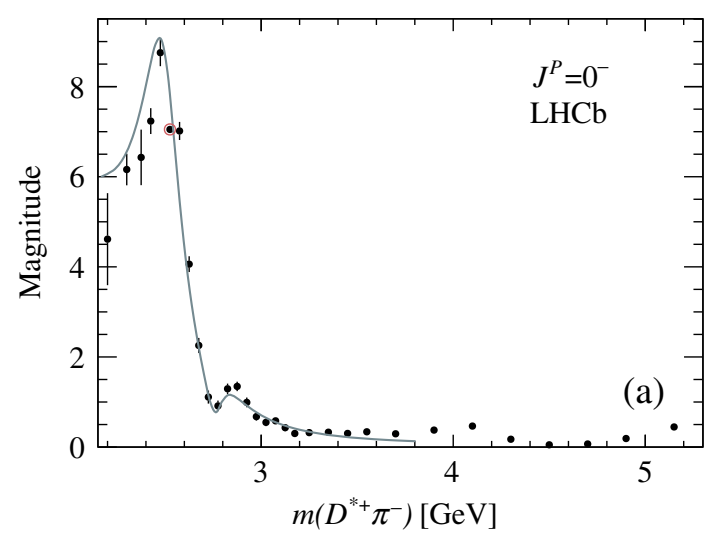

Figure 9 shows the fitted magnitude and phase of the $1^{+} S$ amplitude. The presence of a broad structure can be noted close to threshold with a corresponding phase motion as expected for a resonance. The magnitude and phase show further activity in the $2.8 \mathrm{GeV}$ mass region, suggesting the presence of an additional $1^{+} S$ resonance. However, the introduction of a new $1^{+} S$ Breit-Wigner resonance with floating parameters in that mass region does not produce a significant contribution. The highmass enhancement in the amplitude, on the other hand, is due to symmetrization effects due to the presence of two identical pions.

The $J^{P}=0^{-}$QMI magnitude and phase are shown in Fig. 10. In addition to the $D_{0}(2550)$ resonance, further activity can be seen in the $2.8 \mathrm{GeV}$ mass region both in amplitude and phase, suggesting the presence of a possible new excited $D_{0}^{\prime}$ resonance. The $J^{P}=0^{-}$amplitude and phase distributions are fitted using the model

$$
\begin{aligned}
C(m)= & p s(m) e^{-a m}+c_{1} \mathrm{BW}_{D_{0}}\left(m, m_{0}, \Gamma_{0}\right) e^{i \alpha} \\
& +c_{2} \mathrm{BW}_{D_{0}^{\prime}}\left(m, m_{1}, \Gamma_{1}\right) e^{i \beta},
\end{aligned}
$$

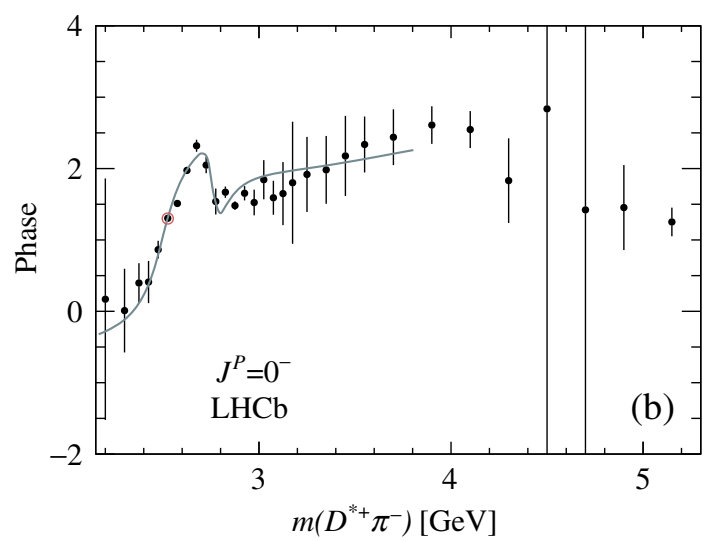

FIG. 10. (a) Magnitude and (b) phase of the $J^{P}=0^{-}$amplitude from the QMI method. The red circle indicates the mass bin where the complex amplitude has been fixed. The curves are the result from the fit described in the text. 
where $p s(m)$ is the $D^{*+}$ momentum in the $D^{*+} \pi^{-}$centerof-mass frame and $a, c_{1}, c_{2}, \alpha$ and $\beta$ are free parameters. The parameters of the $D_{0}(2550)$ resonance $\left(m_{0}, \Gamma_{0}\right)$ are fixed to the values extracted from the amplitude analysis (see Table II), while the parameters of the $D_{0}^{\prime}$ resonance, $\left(m_{1}, \Gamma_{1}\right)$, are free. The first term in the above equation represents a threshold $J^{P}=0^{-}$nonresonant term. The fit is performed in terms of real and imaginary parts of the amplitude and then converted into amplitude and phase when projected on the data in Fig. 10. The $D_{0}^{\prime}$ fitted parameters are

$m\left(D_{0}^{\prime}\right)=2782 \pm 13 \mathrm{MeV}, \quad \Gamma\left(D_{0}^{\prime}\right)=146 \pm 23 \mathrm{MeV}$,

and the significance, computed as the ratio between the fitted fraction divided by the statistical uncertainty, is $3.2 \sigma$. However, an attempt to include this new possible resonance in the amplitude analysis gives a fraction consistent with zero.

To search for additional states, the QMI method is used for the most significant amplitudes, i.e., those with $J^{P}=1^{-}$
(Fig. 11), $J^{P}=1^{+} D$ (Fig. 12), and $J^{P}=2^{+}$(Fig. 13). In mass regions where the amplitude is consistent with zero the phase is not well measured and therefore statistical uncertainties are large. Superimposed on the QMI amplitudes are the Breit-Wigner functions, with arbitrary normalizations, describing the $D_{1}^{*}(2600)$ (Fig. 11) and $D_{1}(2420)$ resonances (Fig. 12), respectively, using the fitted parameters given in Table II. Similarly, the $J^{P}=2^{+}$amplitude is shown in Fig. 13 with $D_{2}^{*}(2460)$ resonance parameters fixed to the values reported in Ref. [3].

A good agreement between the results from the QMI method and the expected line shape of the Breit-Wigner description of the resonances is found. In the case of the $J^{P}=1^{-}$amplitude no additional structure can be seen, and the enhancement at high mass can be associated to the reflection due to the presence of two identical $\pi^{-}$mesons. Some amplitudes as $J^{P}=1^{+} D, J^{P}=2^{+}$and $J^{P}=1^{-}$ evidence some points off from the Breit-Wigner behavior in the threshold region. Since in these regions phase space is limited, these effects can be due to cross-feeds from other partial waves.
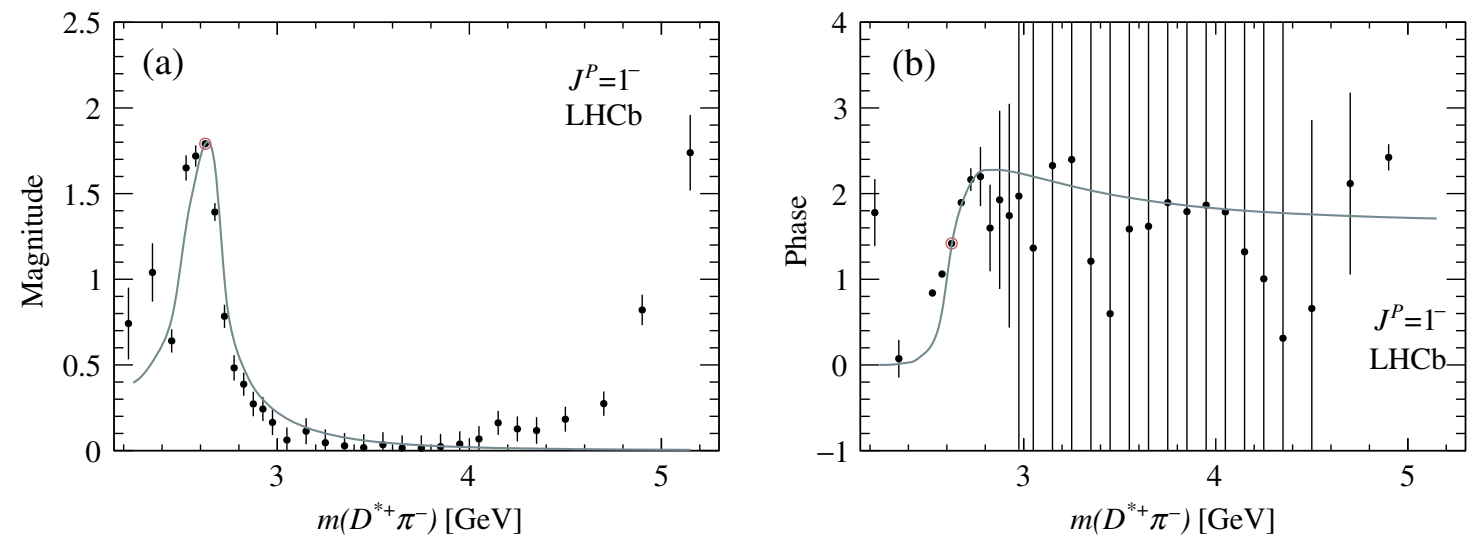

FIG. 11. (a) Magnitude and (b) phase of the $J^{P}=1^{-}$amplitude from the QMI method. The red circle indicates the mass bin where the complex amplitude has been fixed. The curves represent the Breit-Wigner function describing the $D_{1}^{*}(2600)$ resonance.
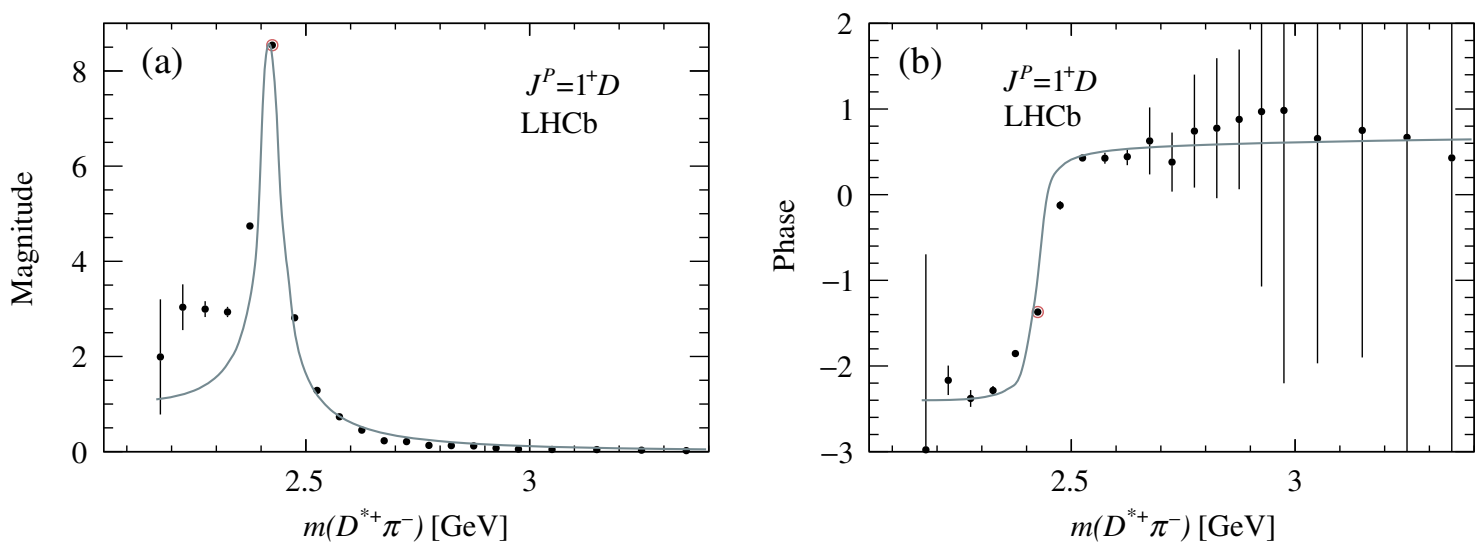

FIG. 12. (a) Magnitude and (b) phase of the $J^{P}=1^{+} D$ amplitude from the QMI method. The red circle indicates the mass bin where the complex amplitude has been fixed. The curves represent the Breit-Wigner function describing the $D_{1}(2420)$ resonance. 

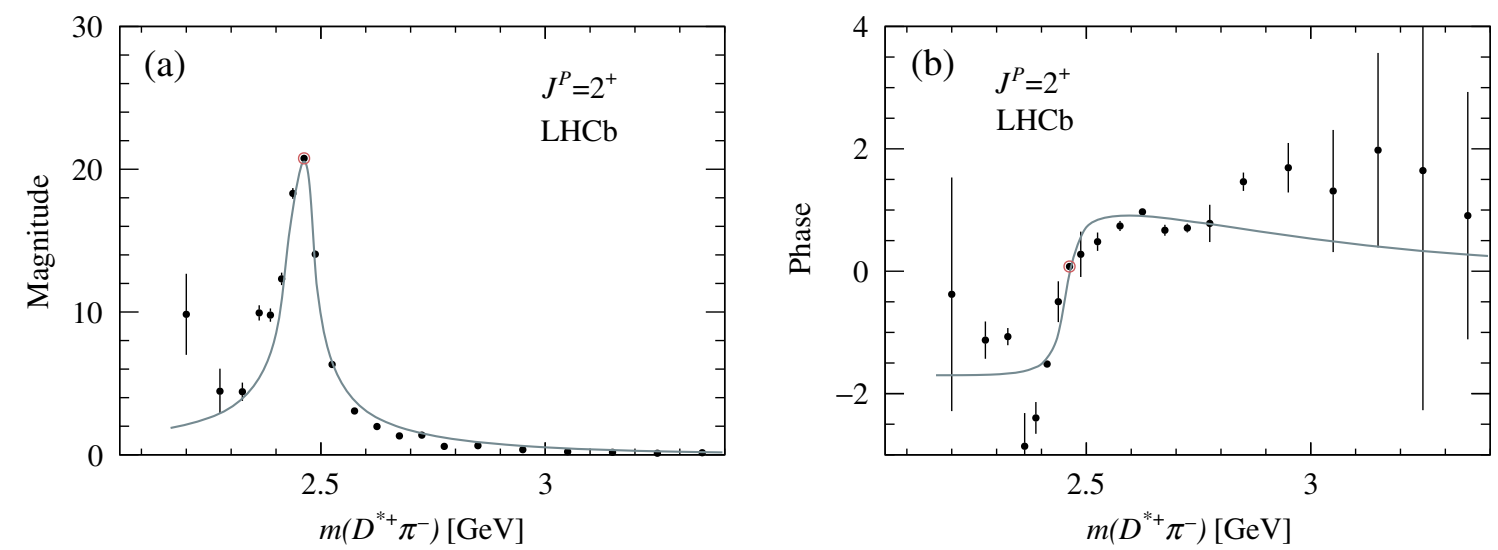

FIG. 13. (a) Magnitude and (b) phase of the $J^{P}=2^{+}$amplitude from the QMI method. The red circle indicates the mass bin where the complex amplitude has been fixed. The curves represent the Breit-Wigner function describing the $D_{2}^{*}(2460)$ resonance.

\section{FIT RESULTS}

The data are fitted using three different models described below.

(i) The $J^{P}=1^{+} S$ and $J^{P}=0^{-}$are described by QMI amplitudes. This model gives the best description of the data and is used to search for new states and obtain the Breit-Wigner parameters for several resonances.

(ii) All the amplitudes are described by relativistic Breit-Wigner functions. This model is used to obtain Breit-Wigner parameters for the $D_{1}(2430)$ and $D_{0}(2550)$ resonances and measure the partial branching fractions for $\mathcal{B}\left(B^{-} \rightarrow R^{0} \pi^{-}\right)$, where $R^{0}$ indicates the charmed meson intermediate state.

(iii) Mixing is allowed between the $1^{+}$amplitudes. This model allows to measure the $D_{1}$ and $D_{1}^{\prime}$ Breit-Wigner parameters and their mixing angle and phase.

\section{A. Results from the QMI model}

In this fitting model the $J^{P}=1^{+} S$ and $J^{P}=0^{-}$are described by QMI, while all the other amplitudes are described by relativistic Breit-Wigner functions with parameters given in Table II. The results from the fit are given in Table III. The dominance of the $D_{1}(2420)\left(1^{+} D\right)$ resonance can be noted, with important contributions from $1^{+} S$ QMI and $D_{2}^{*}(2460)$ amplitudes. The sum of fractions is larger than $100 \%$, indicating important interference effects.

The fit projections for Run 2 data (not biased by the $\pi^{+} \pi^{-} \pi^{-}$mass cut) are shown in Fig. 14. Figure 15 shows the fit projections on $m\left(D^{*+} \pi^{-}\right)_{\text {low }}$ using the total dataset together with all the contributing amplitudes and the significant interference contributions. Using statistical uncertainties only, the separate fits for Run 1 and Run 2 give $\chi^{2} / \mathrm{ndf}=2348 / 1748=1.34$ and $\chi^{2} / \mathrm{ndf}=2111 /$ $1780=1.19$, respectively. For a fit to the total dataset $\chi^{2} /$ ndf $=2551 / 1784=1.43$. However it has to be taken into account that in this fit the total sample size is double and therefore statistical uncertainties are smaller. These $\chi^{2} /$ ndf values indicate a good description of Run 2 data, but a worse description of the Run 1 data indicating some limitation in the handling of the efficiency for this dataset.

\section{Systematic uncertainties}

Systematic uncertainties reported in Table II and alternative fit models (described later), whose results are given in Tables IX and X, are evaluated as follows. When multiple contributions are needed to describe a given effect, the average value of the absolute deviations from the reference fit is taken as a systematic uncertainty.

Table IV gives details on the contributions to the systematic uncertainties on the fractions and phases for the model where the $J^{P}=1^{+} S$ and $J^{P}=0^{-}$amplitudes are described by QMI. The effect of the background (labeled as Purity) is studied by changing the selection cut corresponding to lower (with $\mathcal{R}>1.1, p=0.92,66064$ candidates) or higher (with $\mathcal{R}>0.2, p=0.87,85466$ candidates) purity. The contribution due to the description of the

TABLE III. Fit results from the amplitude analysis for the model where the $J^{P}=1^{+} S$ and $J^{P}=0^{-}$amplitudes are described by QMI. The first uncertainty is statistical, the second systematic.

\begin{tabular}{lrcc}
\hline \hline Resonance & $J^{P}$ & Fraction $(\%)$ & Phase (rad) \\
\hline$D_{1}(2420)$ & $1^{+} D$ & $59.8 \pm 0.3 \pm 2.9$ & 0 \\
$1^{+} S$ QMI & $1^{+} S$ & $28.3 \pm 0.3 \pm 1.9$ & $-1.19 \pm 0.01 \pm 0.15$ \\
$D_{2}^{*}(2460)$ & $2^{+}$ & $15.3 \pm 0.2 \pm 0.3$ & $-0.71 \pm 0.01 \pm 0.48$ \\
$D_{1}(2420)$ & $1^{+} S$ & $2.8 \pm 0.2 \pm 0.5$ & $1.43 \pm 0.02 \pm 0.31$ \\
$0^{-}$QMI & $0^{-}$ & $10.6 \pm 0.2 \pm 0.7$ & $1.94 \pm 0.01 \pm 0.19$ \\
$D_{1}^{*}(2600)$ & $1^{-}$ & $6.0 \pm 0.1 \pm 0.6$ & $1.20 \pm 0.02 \pm 0.05$ \\
$D_{2}(2740)$ & $2^{-} P$ & $1.9 \pm 0.1 \pm 0.4$ & $-1.57 \pm 0.04 \pm 0.15$ \\
$D_{2}(2740)$ & $2^{-} F$ & $3.2 \pm 0.2 \pm 1.1$ & $1.11 \pm 0.04 \pm 0.29$ \\
$D_{3}^{*}(2750)$ & $3^{-}$ & $0.35 \pm 0.04 \pm 0.05$ & $-1.17 \pm 0.07 \pm 0.31$ \\
Sum & & $128.2 \pm 0.6 \pm 3.8$ & \\
\hline \hline
\end{tabular}



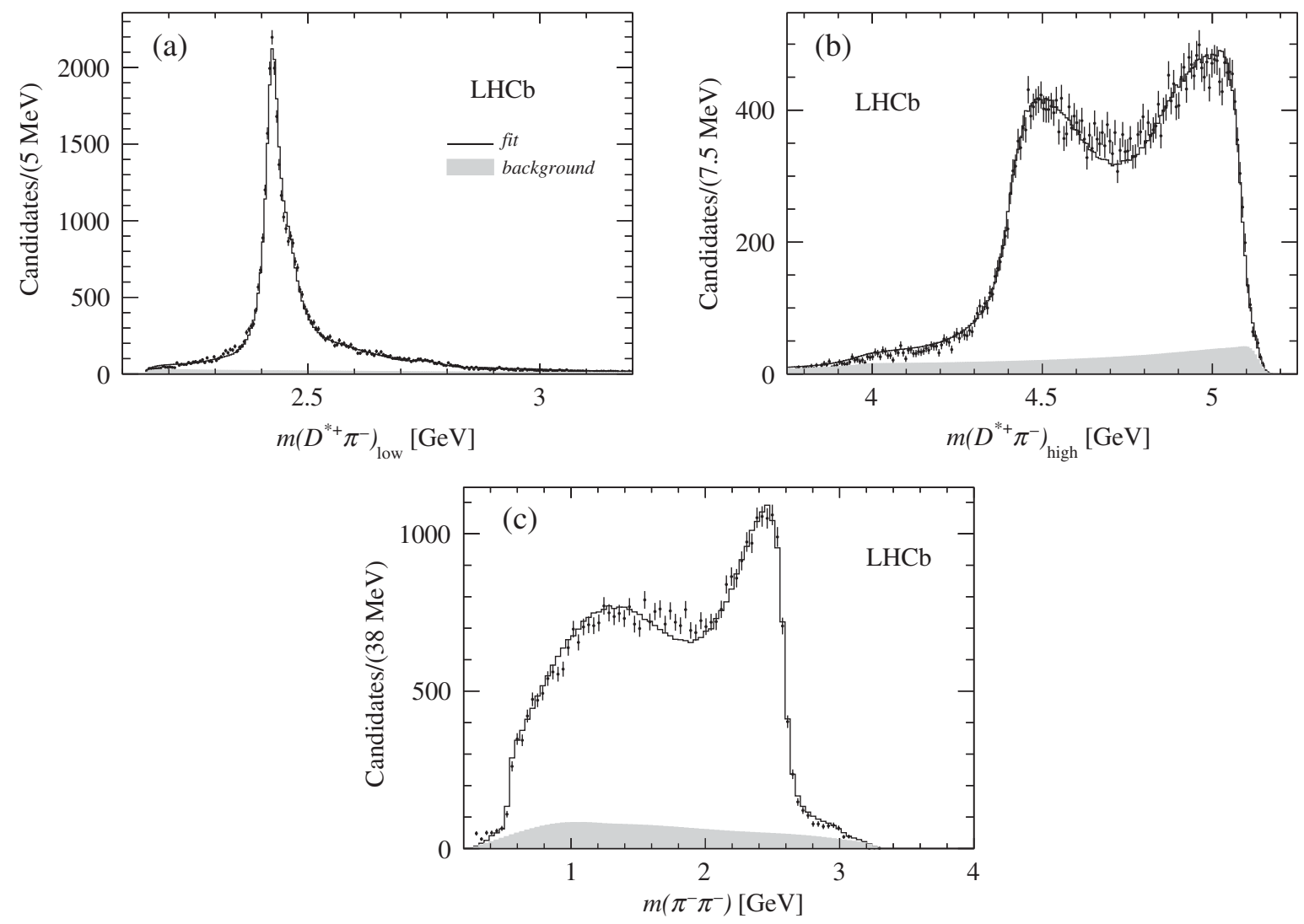

FIG. 14. Projections of the fit to Run 2 data from the QMI fitting model. The background contribution is shown in gray. Data are represented with filled dots and the line is the results from the fit.

resonance model (labeled as BW) is estimated by varying the Blatt-Weisskopf radius between 1 and $5 \mathrm{GeV}^{-1}$. The effect of the uncertainty on the resonance parameters is estimated by varying their values within uncertainties. The label Res.(a) indicates a variation of the parameters of a given resonance, Res.(b) indicates a variation of the parameters of all the other resonances contributing to the $B^{-}$decay. The effect of the uncertainty of the background size (labeled as Bkg size) is estimated by modifying the value of the fixed purity value in the fit $(90 \%)$ by $\pm 0.5 \%$. The effect of the small discrepancy between the data and fit projections on the $\cos \theta$ and $\cos \theta_{H}$ distribution (labeled as Data/sim) is evaluated by weighting the efficiency distribution to match the data. The effect of the limited simulation sample (labeled as Sim) is evaluated by fitting the data using 100 binned 2-dimensional efficiency tables obtained from the reference one through Poisson fluctuations of the entries in each bin. Virtual contributions such as $B^{-} \rightarrow B_{v}^{* 0} \pi^{-}$[14] (labeled as Mod) are included and excluded in the fit. The root-mean-square value of the deviations of the fraction from the reference fit are taken as systematic uncertainties. All the different contributions are added in quadrature. The dominant sources of systematic uncertainties are found to be due to the Blatt-Weisskopf radius.
Table V gives details on the contributions to the systematic uncertainties for the measured masses and widths of the resonances contributing to the $B^{-}$decay. In this case only the most relevant contributions are listed. From the study of large control samples, a systematic uncertainty of $0.0015 Q$ on the mass scale is added, where $Q$ is the $Q$-value involved in the resonance decay.

The consistency between the Run 1 and Run 2 datasets is tested performing separate fits to the data and good agreement is obtained, within the uncertainties, on fractions and relative phases. Separate fits are performed to subsamples of the data where the $D^{*+} \pi^{-} \pi^{-}$final state is directly (69\%) or indirectly $(31 \%)$ selected by the trigger conditions. The fitted fractions and phases are found consistent within the statistical uncertainties. A test is performed by weighting the simulated $p_{\mathrm{T}}$ distribution to match the data and recomputing the efficiencies. The impact on the fitted fractions and phases is found to be negligible.

\section{Legendre polynomial moments projections}

A more detailed understanding of the resonant structures present in the $D^{*+} \pi^{-}$mass spectrum and of the agreement between data and fitting model is obtained by looking at the angular distributions as functions of $\cos \theta, \cos \theta_{H}$ and $\cos \gamma$. This is obtained by weighting the $D^{*+} \pi^{-}$mass spectrum by 

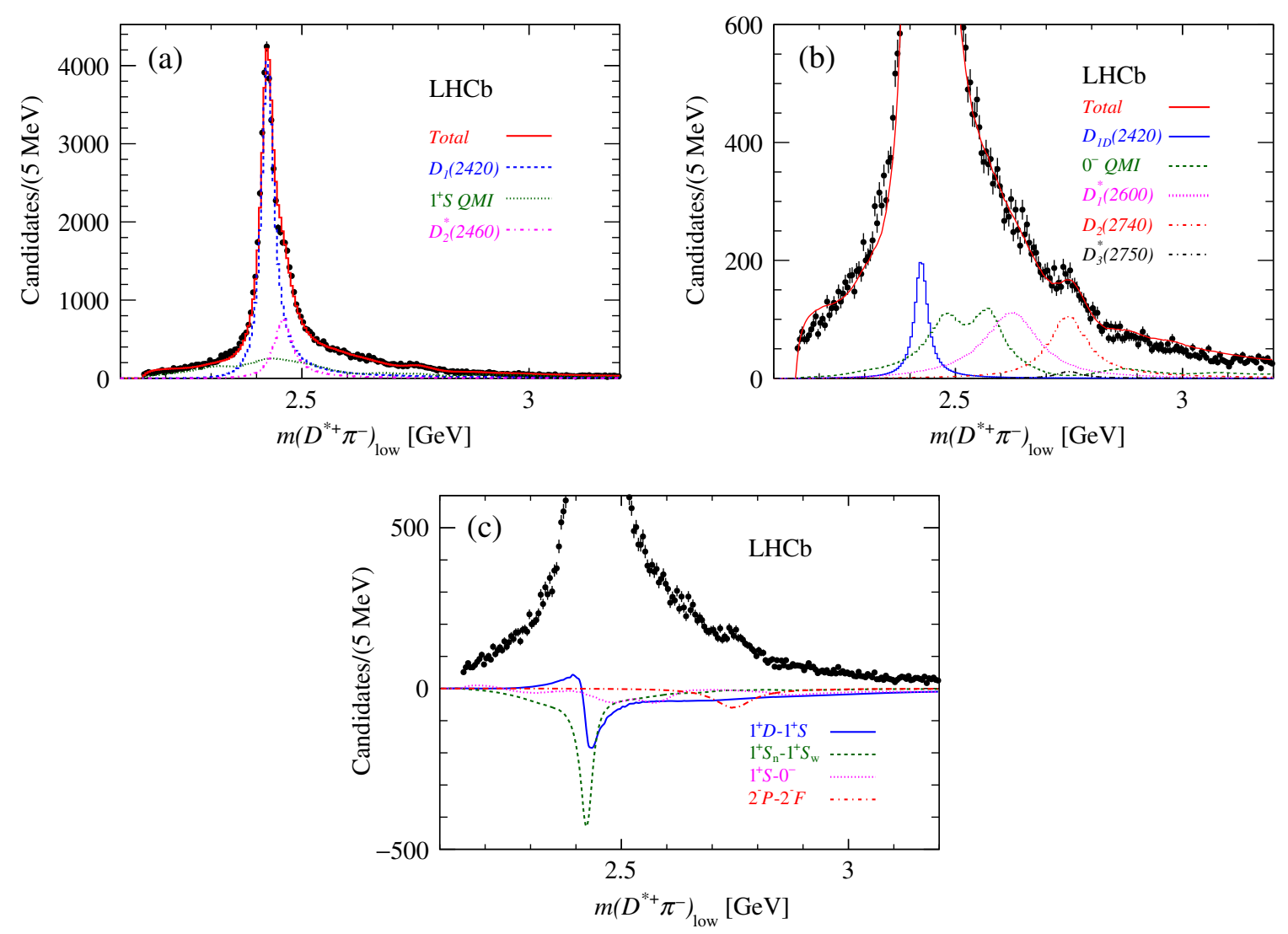

FIG. 15. Projections of the fit to the total dataset from the QMI fitting model with $(a, b)$ all amplitude contributions and (c) the significant interference terms.

the Legendre polynomial moments computed as functions of the above three angles. The $D^{*+} \pi^{-}$mass spectrum weighted by Legendre polynomial moments expressed as functions of $\cos \theta$ is shown in Fig. 16 for $L$ between 1 and 6 and reveals a rich structure. Higher moments are consistent with zero. Equations (15) relate the moments with orbital angular momentum between the $D^{*+}$ and $\pi^{-}$mesons, assuming only partial waves between $L=0$ and $L=3$. Here $S, P, D$ and $F$ indicate the magnitudes of the amplitudes with angular momenta $L=0,1,2,3$ and $\phi$ denotes their relative phases.

$$
\begin{aligned}
& \sqrt{4 \pi}\left\langle Y_{0}^{0}\right\rangle=S^{2}+P^{2}+D^{2}+F^{2} \\
& \sqrt{4 \pi}\left\langle Y_{1}^{0}\right\rangle=2 S P \cos \phi_{S P}+1.789 P D \cos \phi_{P D}+1.757 D F \cos \phi_{D F} \\
& \sqrt{4 \pi}\left\langle Y_{2}^{0}\right\rangle=2 S D \cos \phi_{S D}+0.894 P^{2}+1.757 P F \cos \phi_{P F}+0.639 D^{2}+0.596 F^{2} \\
& \sqrt{4 \pi}\left\langle Y_{3}^{0}\right\rangle=2 S F \cos \phi_{S F}+1.757 P D \cos \phi_{P D}+1.193 D F \cos \phi_{D F} \\
& \sqrt{4 \pi}\left\langle Y_{4}^{0}\right\rangle=1.746 P F \cos \phi_{P F}+0.857 D^{2}+0.545 F^{2} \\
& \sqrt{4 \pi}\left\langle Y_{5}^{0}\right\rangle=1.699 D F \cos \phi_{D F} \\
& \sqrt{4 \pi}\left\langle Y_{6}^{0}\right\rangle=0.840 F^{2}
\end{aligned}
$$

A comparison with Table I allows for the identification of the resonant contributions to each distribution, listed in Table VI.

Significant interference effects between $1^{+}$amplitudes can be observed in the $Y_{2}^{0}$ distribution and a clean $2^{+}$signal due to $D_{2}^{*}(2460)$ can be seen in the $Y_{4}^{0}$ distribution. Other moments show rather complex structures. An overall good description of the data is obtained, although some small discrepancy can be seen in $Y_{3}^{0}$ and $Y_{5}^{0}$. This is expected, given the large number of physical contributions 
TABLE IV. Absolute systematic uncertainties on the fractions (in \%) (top), and phases (bottom) for the model where the $J^{P}=1^{+} S$ and $J^{P}=0^{-}$amplitudes are described by QMI.

\begin{tabular}{lcccccccccc}
\hline \hline Resonance & $J^{P}$ & Purity & BW & Res.(a) & Res.(b) & Bkg size & Data/sim & Sim & Mod & Total \\
\hline$D_{1}(2420)$ & $1^{+} D$ & 0.36 & 2.88 & 0.05 & 0.01 & 0.30 & 0.19 & 0.33 & 0.12 & 2.9 \\
$1^{+} S$ QMI & $1^{+} S$ & 0.54 & 1.37 & & 0.01 & 0.27 & 0.16 & 0.34 & 1.17 & 1.9 \\
$D_{2}^{*}(2460)$ & $2^{+}$ & 0.14 & 0.04 & 0.05 & 0.01 & 0.05 & 0.04 & 0.26 & 0.00 & 0.3 \\
$D_{1}(2420)$ & $1^{+} S$ & 0.03 & 0.46 & 0.02 & 0.01 & 0.01 & 0.02 & 0.21 & 0.00 & 0.5 \\
$0^{-}$QMI & $0^{-}$ & 0.07 & 0.08 & & 0.01 & 0.03 & 0.07 & 0.17 & 0.69 & 0.72 \\
$D_{1}^{*}(2600)$ & $1^{-}$ & 0.05 & 0.53 & 0.01 & 0.01 & 0.02 & 0.06 & 0.14 & 0.04 & 0.6 \\
$D_{2}(2740)$ & $2^{-} P$ & 0.11 & 0.36 & 0.01 & 0.00 & 0.06 & 0.06 & 0.10 & 0.07 & 0.4 \\
$D_{2}(2740)$ & $2^{-} F$ & 0.09 & 1.13 & 0.02 & 0.01 & 0.12 & 0.07 & 0.12 & 0.29 & 1.1 \\
$D_{3}^{*}(2750)$ & $3^{-}$ & 0.02 & 0.02 & 0.00 & 0.00 & 0.01 & 0.02 & 0.03 & 0.008 & 0.1 \\
$1^{+} S$ QMI & $1^{+} S$ & 0.01 & 0.15 & & 0.00 & 0.00 & 0.00 & 0.00 & 0.010 & 0.15 \\
$D_{2}^{*}(2460)$ & $2^{+}$ & 0.01 & 0.48 & 0.01 & 0.00 & 0.00 & 0.00 & 0.01 & 0.01 & 0.48 \\
$D_{1}(2420)$ & $1^{+} S$ & 0.02 & 0.31 & 0.00 & 0.00 & 0.01 & 0.02 & 0.03 & 0.00 & 0.31 \\
$0^{-}$QMI & $0^{-}$ & 0.01 & 0.08 & 0.00 & 0.01 & 0.17 & 0.00 & 0.01 & 0.04 & 0.19 \\
$D_{1}^{*}(2600)$ & $1^{-}$ & 0.03 & 0.03 & 0.01 & 0.00 & 0.01 & 0.00 & 0.02 & 0.02 & 0.05 \\
$D_{2}(2740)$ & $2^{-} P$ & 0.04 & 0.14 & 0.02 & 0.00 & 0.00 & 0.01 & 0.04 & 0.02 & 0.15 \\
$D_{2}(2740)$ & $2^{-} F$ & 0.06 & 0.28 & 0.02 & 0.00 & 0.01 & 0.00 & 0.03 & 0.02 & 0.29 \\
$D_{3}^{*}(2750)$ & $3^{-}$ & 0.13 & 0.28 & 0.04 & 0.00 & 0.01 & 0.03 & 0.04 & 0.04 & 0.31 \\
\hline \hline
\end{tabular}

(see Table VI) to the shape of the $Y_{L}^{0}$ moments which are also sensitive to efficiency effects.

Additional information can be obtained from the fit projections on the $D^{*+} \pi^{-}$mass spectrum weighted by Legendre polynomial moments computed as functions of $\cos \theta_{H}$ (labeled as $Y_{L}^{H}$ ) and $\cos \gamma$ (labeled as $Y_{L}^{\gamma}$ ), shown in Fig. 17(a) and Fig. 17(b), respectively. The two $Y_{2}^{H}\left(\cos \theta_{H}\right)$ and $Y_{2}^{\gamma}(\cos \gamma)$ projections show interference effects between the $D_{1}(2420)$ and $D_{2}^{*}(2460)$ resonances. The $Y_{2}^{\gamma}$ distribution also shows an enhancement at the position of the $D_{1}^{*}(2600)$ resonance. Other moments are consistent with zero.

\section{Search for additional contributions and spin-parity determination}

The presence of additional contributions is tested by adding them to the reference fit using the total dataset. The significance of each contribution is computed as its fitted fraction divided by its statistical uncertainty. No evidence is found for the $D_{1}^{*}(2760)$ or $D_{2}^{*}(3000)$ contributions, previously observed in the $B^{-} \rightarrow D^{+} \pi^{-} \pi^{-}$decay [14]. Their statistical significance is found to be 2.4 and 0.0 , respectively. Virtual contributions, as described in Ref. [14], are found to be small with a statistical significance of $4.4 \sigma$ but ignored because they have a small fraction $(0.12 \pm 0.03) \%$, an uncertain physical meaning [14] and do not significantly improve the fit $\chi^{2}$.

The presence of a $D^{0} \pi^{+} \pi^{-} \pi^{-}$nonresonant contribution has been tested but excluded from the final fit. Its effect, due to the presence of broad $J^{P}=1^{+}$resonances, is to produce large interference effects so that the total fraction increases to large and rather unphysical values without significantly improving the fit quality.
It has been noted that the QMI $1^{+} S$ amplitude (Fig. 9) shows activity both in amplitude and phase in the mass region around $2.8 \mathrm{GeV}$ which could correspond to the presence of an additional $D_{1}$ resonance. A test is performed including an additional $1^{+} S$ Breit-Wigner resonance in this mass region with free parameters. However, no significant contribution for this additional state is found.

The QMI approach is used for the most significant amplitudes and Breit-Wigner behavior is obtained for $J^{P}=1^{+}, J^{P}=2^{+}, J^{P}=0^{-}$and $J^{P}=1^{-}$resonances. For other contributions, such as the $J^{P}=2^{-} D_{2}(2740)$ or $J^{P}=3^{-} D_{3}^{*}(2750)$ resonances, this is not possible due to the weakness of these contributions. For these two states, a spin analysis is performed. For each state additional fits are performed where the masses and widths are fixed to the

TABLE V. Systematic uncertainty contributions to the measured mass and width (in $\mathrm{MeV}$ ) of the different resonances contributing to the $B^{-}$decay.

\begin{tabular}{lcrccr}
\hline \hline Resonance & Parameter & BW & Purity & Mass scale & Total \\
\hline$D_{1}(2420)$ & Mass & 0.6 & 0.1 & 0.4 & 0.7 \\
& Width & 2.7 & 0.4 & & 2.7 \\
$D_{0}(2550)$ & Mass & 3.2 & 6.7 & 0.6 & 7.4 \\
& Width & 14.7 & 8.1 & & 16.8 \\
$D_{1}^{*}(2600)$ & Mass & 2.9 & 2.9 & 0.7 & 4.5 \\
& Width & 14.9 & 12.7 & & 19.6 \\
$D_{2}(2740)$ & Mass & 4.3 & 5.6 & 0.9 & 7.1 \\
& Width & 25.1 & 8.0 & & 26.3 \\
$D_{3}^{*}(2750)$ & Mass & 5.8 & & 0.9 & 5.9 \\
& Width & 14.4 & & 0.4 & 14.4 \\
$D_{1}(2430)$ & Mass & 7.0 & 5.5 & 0.4 & 8.9 \\
& Width & 14.0 & 24.0 & & 27.8 \\
\hline \hline
\end{tabular}



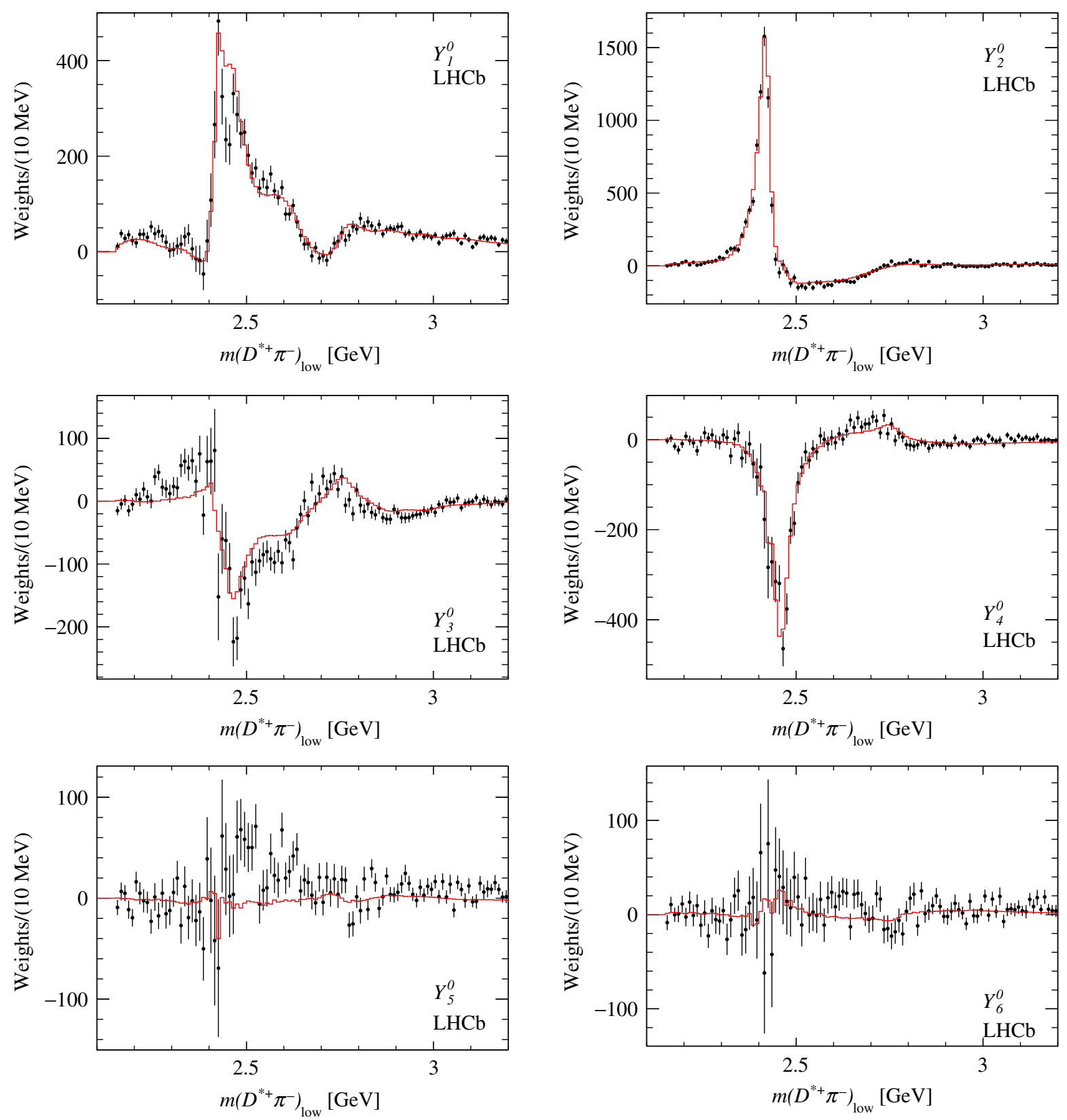

FIG. 16. Legendre polynomial moments $Y_{L}^{0}(\cos \theta)$ as functions of $m\left(D^{*+} \pi^{-}\right)$for Run 2 data. The data are represented by filled dots and the superimposed histograms result from the amplitude analysis described in the text.

results given in Table II but where the angular distributions are replaced by those from other possible spin assignments. For the $D_{2}(2740)$ resonance, $J^{P}=0^{-}, 1^{+} D, 1^{+} S, 1^{-}, 2^{+}$ are tried but the likelihood and $\chi^{2}$ variations exclude all the alternative hypotheses with significances greater than $8 \sigma$. The estimate of the significance is obtained using $\sqrt{\Delta \chi^{2}}$, where $\Delta \chi^{2}$ is the variation of the fit $\chi^{2}$ for the given spin hypothesis. Similarly, for the $D_{3}^{*}(2750)$ resonance, values of $J^{P}=0^{-}, 1^{+} D, 1^{+} S, 1^{-}$and $2^{+}$are tried but excluded with significances greater than $8 \sigma$. In conclusion, the present analysis measures the resonance parameters and establishes the quantum numbers of the $D_{0}(2550)$,
$D_{1}^{*}(2600), D_{2}(2740)$ and $D_{3}^{*}(2750)$ resonances. The fitted parameters are compared with those measured by other analyses or other experiments in Table VII. Note that different methods have been used to extract the resonances parameters. The results from the BABAR [11] and $\mathrm{LHCb}$ [12] collaborations come from inclusive studies of the $D^{+} \pi^{-}, D^{0} \pi^{+}$and $D^{*+} \pi^{-}$combinations where signals are fitted directly on the mass spectra. In the case of the $D^{*+} \pi^{-}$ mass spectrum, resonance production is enhanced by the use of selections on the helicity angle $\theta_{H}$. Cross feeds from the resonance production in the $D^{*+} \pi^{-}$system are present in the $D^{+} \pi^{-}$and $D^{0} \pi^{+}$mass spectra. The LHCb results 
TABLE VI. Relationship between the Legendre polynomial moments $Y_{L}^{0}$ and spin amplitudes. In the column describing the interfering amplitudes, the left side amplitude is intended to interfere with any of the amplitudes listed on the right side.

\begin{tabular}{|c|c|c|c|}
\hline Moment & $\begin{array}{l}\text { Squared } \\
\text { amplitudes }\end{array}$ & $\begin{array}{l}\text { Interfering } \\
\text { amplitudes }\end{array}$ & \\
\hline$Y_{1}^{0}$ & & $\begin{array}{l}1^{+} S \\
0^{-} \\
1^{-} \\
1^{+} D \\
2^{+}\end{array}$ & $\begin{array}{l}0^{-}, 1^{-}, 2^{-} P \\
1^{+} D, 2^{+} \\
1^{+} D \\
2^{-} P, 2^{-} F, 3^{-} \\
3^{-}, 2^{-} F\end{array}$ \\
\hline$Y_{2}^{0}$ & $\begin{array}{l}0^{-}, 1^{-}, 2^{-} P \\
\quad 1^{+} D, 2^{+}, 3^{-}\end{array}$ & $\begin{array}{l}1^{+} S \\
3^{-} \\
2^{-} F\end{array}$ & $\begin{array}{l}1^{+} D, 2^{+} \\
0^{-}, 1^{-}, 2^{-} P \\
0^{-}, 1^{-}, 2^{-} P\end{array}$ \\
\hline$Y_{3}^{0}$ & & $\begin{array}{l}1^{+} S \\
1^{+} D \\
2^{+}\end{array}$ & $\begin{array}{l}3^{-}, 2^{-} F \\
0^{-}, 1^{-}, 2^{-} P, 3^{-}, 2^{-} F \\
0^{+}, 1^{-}, 3^{-}, 2^{-} F\end{array}$ \\
\hline$Y_{4}^{0}$ & $1^{+} D, 2^{+}$ & $\begin{array}{l}3^{-} \\
2^{-} F\end{array}$ & $\begin{array}{l}0^{-}, 2^{-} \\
0^{-}, 1^{-}, 2^{-} P\end{array}$ \\
\hline$Y_{5}^{0}$ & & $\begin{array}{l}3^{-} \\
2^{-} F\end{array}$ & $\begin{array}{l}1^{+} D, 2^{+} \\
1^{+} D, 2^{+}\end{array}$ \\
\hline$Y_{6}^{0}$ & $3^{-}, 2^{-} F$ & & \\
\hline
\end{tabular}

from Ref. [13,14], on the other hand, come from Dalitz plot analyses of $B$ decays.

\section{B. Results from the Breit-Wigner model}

Table II gives the resonance parameters for the $D_{1}(2430)$ and $D_{0}(2550)$ states when they are described by relativistic Breit-Wigner functions. An amplitude analysis performed using this model gives the results shown in Table VIII. In this case $\chi^{2} / \mathrm{ndf}=2348 / 1748=1.34$ and $\chi^{2} / \mathrm{ndf}=$ $2205 / 1780=1.24$ for Run 1 and Run 2 data, respectively. Somewhat reduced fractional contributions from the $D_{1}(2430)$ and $D_{0}(2550)$ resonances with respect to the

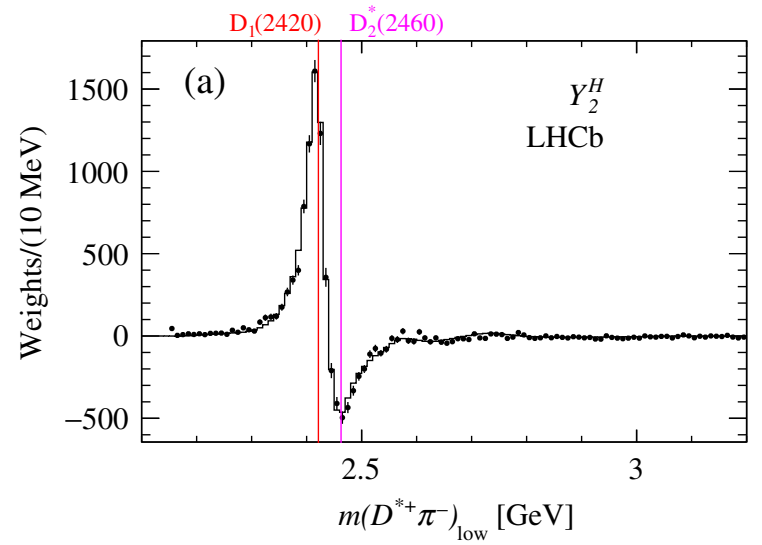

QMI approach can be seen. This effect can be understood since in this model the $J^{P}=1^{+} S$ and $J^{P}=0^{-}$contributions do not include possible additional contributions from higher mass resonances. Systematic uncertainties are evaluated as described in Sec. VII.

\section{Results from the $J^{P}=1^{+}$mixing model}

A consequence of the heavy-quark symmetry is that, in the infinite-mass heavy quark limit, heavy-light $Q \bar{q}$ mesons can be classified in doublets labeled by the value of the total angular momentum $j_{q}$ of the light degrees of freedom with respect to the heavy quark $Q$ [33]. In the quark model $\boldsymbol{j}_{q}$ would be given by $\boldsymbol{j}_{q}=\boldsymbol{s}_{q}+\boldsymbol{L}$, where $\boldsymbol{L}$ is the light quark orbital angular momentum. The Heavy Quark Effective Theory predicts that the two $J^{P}=1^{+}$mesons, with $j_{q}=\frac{1}{2}$ and $j_{q}=\frac{3}{2}$, decay into the $D^{*} \pi$ final state via the $S$ - and $D$-wave, respectively. Due to the finite $c$-quark mass, the observed physical $1^{+}$states can be a mixture of such pure states. The mixing can occur for instance via the common $D^{*} \pi$ decay channel and the resulting $D_{1}^{\prime}$ and $D_{1}$ amplitudes are a superposition of the $S$ - and $D$-wave amplitudes

$$
\begin{aligned}
& A^{D_{1}^{\prime}}=A^{1 S} \cos \omega-A^{1 D} \sin \omega e^{i \psi}, \\
& A^{D_{1}}=A^{1 S} \sin \omega+A^{1 D} \cos \omega e^{-i \psi},
\end{aligned}
$$

where $\omega$ is the mixing angle and $\psi$ is a complex phase.

In this model the $J^{P}=1^{+} D_{1}^{\prime}$ amplitude is taken as reference. The $J^{P}=1^{+} S$ amplitudes are described by relativistic Breit-Wigner functions with free parameters, while the $J^{P}=0^{-}$amplitude is described by the QMI model. All the other resonances are described by relativistic Breit-Wigner functions with parameters fixed to the values reported in Table II. Table IX gives details on the fractions and relative phases.

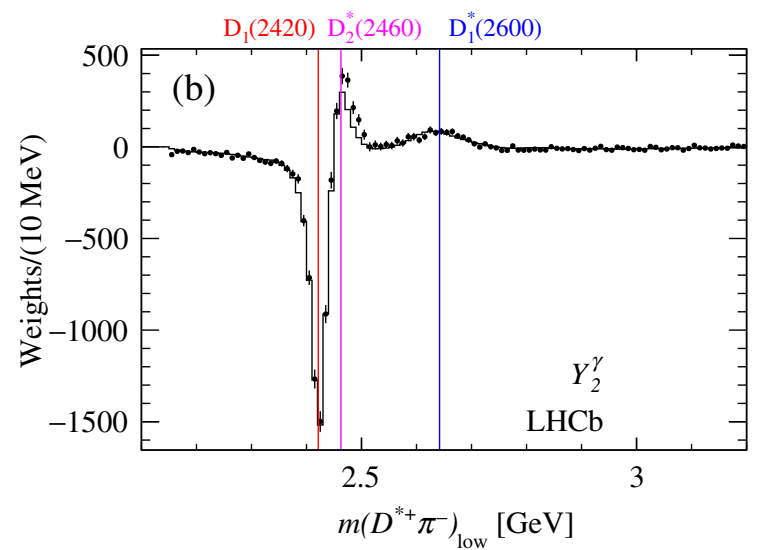

FIG. 17. Distributions of the Legendre polynomial moments (a) $Y_{2}^{H}\left(\cos \theta_{H}\right)$ and (b) $Y_{2}^{\gamma}(\cos \gamma)$ as a function of $m\left(D^{*+} \pi^{-}\right)$for Run 2 data. The data are represented by filled dots while the superimposed histograms result from the amplitude analysis described in the text. The vertical lines indicate the positions of the $D_{1}(2420), D_{2}^{*}(2460)$ and $D_{1}^{*}(2600)$ resonances. 
TABLE VII. Comparison of the resonance parameters measured in the present work with previous measurements. The first uncertainty is statistical, the second systematic.

\begin{tabular}{lccccc}
\hline \hline Resonance & $J^{P}$ & Decays & Mass $[\mathrm{MeV}]$ & Width [MeV] & References \\
\hline$D_{0}(2550)^{0}$ & $0^{-}$ & $D^{*+} \pi^{-}$ & $2518 \pm 2 \pm 7$ & $199 \pm 5 \pm 17$ & This work \\
$D_{J}(2550)^{0}$ & & $D^{*+} \pi^{-}$ & $2539.4 \pm 4.5 \pm 6.8$ & $130 \pm 12 \pm 13$ & $B A B A R[11]$ \\
$D_{J}(2580)^{0}$ & & $D^{*+} \pi^{-}$ & $2579.5 \pm 3.4 \pm 3.5$ & $177.5 \pm 17.8 \pm 46.0$ & LHCb [12] \\
$D_{1}^{*}(2600)^{0}$ & $1^{-}$ & $D^{*+} \pi^{-}$ & $2641.9 \pm 1.8 \pm 4.5$ & $149 \pm 4 \pm 20$ & This work \\
$D_{J}^{*}(2600)^{0}$ & & $D^{+} \pi^{-}$ & $2608.7 \pm 2.4 \pm 2.5$ & $93 \pm 6 \pm 13$ & $B A B A R[11]$ \\
$D_{J}^{*}(2650)^{0}$ & & $D^{*+} \pi^{-}$ & $2649.2 \pm 3.5 \pm 3.5$ & $140.2 \pm 17.1 \pm 18.6$ & LHCb [12] \\
$D_{1}^{*}(2680)^{0}$ & & $D^{+} \pi^{-}$ & $2681.1 \pm 5.6 \pm 4.9$ & $186.7 \pm 8.5 \pm 8.6$ & LHCb [14] \\
$D_{2}(2740)^{0}$ & $2^{-}$ & $D^{*+} \pi^{-}$ & $2751 \pm 3 \pm 7$ & $102 \pm 6 \pm 26$ & This work \\
$D_{J}(2750)^{0}$ & & $D^{*+} \pi^{-}$ & $2752.4 \pm 1.7 \pm 2.7$ & $71 \pm 6 \pm 11$ & $B A B A R[11]$ \\
$D_{J}(2740)^{0}$ & & $D^{*+} \pi^{-}$ & $2737.0 \pm 3.5 \pm 11.2$ & $73.2 \pm 13.4 \pm 25.0$ & LHCb [12] \\
$D_{3}^{*}(2750)^{0}$ & $3^{-}$ & $D^{*+} \pi^{-}$ & $2753 \pm 4 \pm 6$ & $66 \pm 10 \pm 14$ & This work \\
$D_{J}^{*}(2760)^{0}$ & & $D^{*+} \pi^{-}$ & $2761.1 \pm 5.1 \pm 6.5$ & $74.4 \pm 3.4 \pm 37.0$ & LHCb [12] \\
& & $D^{+} \pi^{-}$ & $2760.1 \pm 1.1 \pm 3.7$ & $74.4 \pm 3.4 \pm 19.1$ & LHCb [12] \\
$D_{J}^{*}(2760)^{+}$ & & $D^{+} \pi^{-}$ & $2763.3 \pm 2.3 \pm 2.3$ & $60.9 \pm 5.1 \pm 3.6$ & $B A B A R[11]$ \\
$D_{3}^{*}(2760)^{+}$ & $3^{-}$ & $D^{0} \pi^{+}$ & $2771.7 \pm 1.7 \pm 3.8$ & $66.7 \pm 6.6 \pm 10.5$ & LHCb [12] \\
$D_{3}^{*}(2760)^{0}$ & $3^{-}$ & $D^{0} \pi^{-}$ & $2798 \pm 7 \pm 1$ & $105 \pm 18 \pm 6$ & LHCb [13] \\
\hline \hline
\end{tabular}

The resulting mixing parameters are

$\omega=-0.063 \pm 0.019 \pm 0.004, \quad \psi=-0.29 \pm 0.09 \pm 0.07$

which are consistent with the results from the Belle collaboration [4],

$$
\begin{aligned}
& \omega=-0.10 \pm 0.03 \pm 0.02 \pm 0.02, \\
& \psi=0.05 \pm 0.20 \pm 0.04 \pm 0.06 .
\end{aligned}
$$

Combining statistical and systematic uncertainties the mixing angle deviates from zero by $2.3 \sigma$.

The $\chi^{2} /$ ndf for the fit to the total dataset is $\chi^{2} / \mathrm{ndf}=$ $2739 / 1780=1.54$. Systematic uncertainties on the mixing

TABLE VIII. Fit results from the amplitude analysis for the model where the $D_{1}(2430)$ and $D_{0}(2550)$ resonances are described by relativistic Breit-Wigner functions. The first uncertainty is statistical, the second systematic.

\begin{tabular}{lrcc}
\hline \hline Resonance & $J^{P}$ & Fraction $(\%)$ & Phase $(\mathrm{rad})$ \\
\hline$D_{1}(2420)$ & $1^{+} D$ & $56.5 \pm 0.3 \pm 1.1$ & 0 \\
$D_{1}(2430)$ & $1^{+} S$ & $26.0 \pm 0.4 \pm 1.7$ & $-1.57 \pm 0.02 \pm 0.08$ \\
$D_{2}^{*}(2460)$ & $2^{+}$ & $15.4 \pm 0.2 \pm 0.1$ & $-0.77 \pm 0.01 \pm 0.01$ \\
$D_{1}(2420)$ & $1^{+} S$ & $5.9 \pm 0.5 \pm 2.9$ & $1.69 \pm 0.02 \pm 0.06$ \\
$D_{0}(2550)$ & $0^{-}$ & $5.3 \pm 0.1 \pm 0.5$ & $1.50 \pm 0.02 \pm 0.06$ \\
$D_{1}^{*}(2600)$ & $1^{-}$ & $5.0 \pm 0.1 \pm 0.5$ & $0.76 \pm 0.02 \pm 0.03$ \\
$D_{2}(2740)$ & $2^{-} P$ & $0.57 \pm 0.07 \pm 0.23$ & $-2.14 \pm 0.07 \pm 0.16$ \\
$D_{2}(2740)$ & $2^{-} F$ & $1.9 \pm 0.1 \pm 1.0$ & $0.49 \pm 0.04 \pm 0.40$ \\
$D_{3}^{*}(2750)$ & $3^{-}$ & $0.78 \pm 0.06 \pm 0.13$ & $-1.54 \pm 0.05 \pm 0.04$ \\
Sum & \multicolumn{3}{l}{$117.3 \pm 0.8 \pm 3.8$} \\
\hline \hline
\end{tabular}

parameters, fractional contributions and relative phases are computed as described in Sec. VII. The measured $D_{1}$ and $D_{1}^{\prime}$ masses and widths are reported in Table II.

\section{MEASUREMENT OF THE BRANCHING FRACTIONS}

The known branching fraction of the $B^{-} \rightarrow D^{*+} \pi^{-} \pi^{-}$ decay mode is $\mathcal{B}\left(B^{-} \rightarrow D^{*+} \pi^{-} \pi^{-}\right)=(1.35 \pm 0.22) \times$ $10^{-3}$ [3]. Table $X$ reports the partial branching fractions for the resonances contributing to the total branching fraction. They are obtained multiplying the $B^{-}$branching fraction by the fractional contributions obtained from the amplitude analysis performed using the Breit-Wigner model for all the resonances and reported in Table VIII. For the $D_{1}$ and $D_{1}^{\prime}$ branching fractions the fractional contributions obtained from the mixing model and reported in Table IX are used. Since the uncertainty on the absolute

TABLE IX. Fit results from the mixing model. The first uncertainty is statistical, the second systematic.

\begin{tabular}{lrcc}
\hline \hline Resonance & $J^{P}$ & Fraction $(\%)$ & Phase (rad) \\
\hline$D_{1}$ & $1^{+}$ & $58.9 \pm 0.7 \pm 2.5$ & 0 \\
$D_{1}^{\prime}$ & $1^{+}$ & $21.9 \pm 2.2 \pm 3.0$ & $-1.06 \pm 0.10 \pm 0.05$ \\
$D_{2}^{*}(2460)$ & $2^{+}$ & $14.0 \pm 0.2 \pm 0.3$ & $2.66 \pm 0.09 \pm 0.15$ \\
$0^{-} Q M I$ & $0^{-}$ & $6.5 \pm 0.2 \pm 1.5$ & $2.03 \pm 0.09 \pm 0.28$ \\
$D_{1}^{*}(2600)$ & $1^{-}$ & $4.9 \pm 0.1 \pm 0.5$ & $-2.24 \pm 0.09 \pm 0.11$ \\
$D_{2}(2740)$ & $2^{-} P$ & $0.72 \pm 0.08 \pm 0.30$ & $-2.59 \pm 0.10 \pm 0.53$ \\
$D_{2}(2740)$ & $2^{-} F$ & $2.9 \pm 0.2 \pm 1.1$ & $0.27 \pm 0.09 \pm 0.47$ \\
$D_{3}^{*}(2750)$ & $3^{-}$ & $0.70 \pm 0.05 \pm 0.10$ & $1.54 \pm 0.10 \pm 0.33$ \\
Sum & & $110.4 \pm 2.3 \pm 4.4$ & \\
\hline \hline
\end{tabular}


TABLE X. Summary of the measurements of the branching fractions. The first uncertainty is statistical, the second systematic, the third is due to the uncertainty on the measurement of the $B^{-} \rightarrow D^{*+} \pi^{-} \pi^{-}$absolute branching fraction. A comparison with measurements obtained by the Belle collaboration [4] is shown.

\begin{tabular}{lccc}
\hline \hline & & \multicolumn{2}{c}{$\mathcal{B}\left(B^{-} \rightarrow R^{0} \pi^{-}\right) \times \mathcal{B}\left(R^{0} \rightarrow D^{*+} \pi^{-}\right) \times 10^{-4}$} \\
\cline { 3 - 4 } Resonance & $J^{P}$ & This analysis & Belle collaboration \\
\hline$D_{1}(2420)$ & $1^{+}$ & $8.42 \pm 0.08 \pm 0.40 \pm 1.40$ & \\
$D_{1}(2430)$ & $1^{+} S$ & $3.51 \pm 0.06 \pm 0.23 \pm 0.57$ & $1.8 \pm 0.3 \pm 0.3 \pm 0.2$ \\
$D_{2}^{*}(2460)$ & $2^{+}$ & $2.08 \pm 0.03 \pm 0.14 \pm 0.34$ & \\
$D_{0}(2550)$ & $0^{-}$ & $0.72 \pm 0.01 \pm 0.07 \pm 0.12$ & \\
$D_{1}^{*}(2600)$ & $1^{-}$ & $0.68 \pm 0.01 \pm 0.07 \pm 0.11$ & \\
$D_{2}(2740)$ & $2^{-}$ & $0.33 \pm 0.02 \pm 0.14 \pm 0.05$ & $6.8 \pm 0.7 \pm 1.3 \pm 0.3$ \\
$D_{3}^{*}(2750)$ & $3^{-}$ & $0.11 \pm 0.01 \pm 0.02 \pm 0.02$ & $5.0 \pm 0.4 \pm 1.0 \pm 0.4$ \\
$D_{1}$ & $1^{+}$ & $7.95 \pm 0.09 \pm 0.34 \pm 1.30$ & \\
$D_{1}^{\prime}$ & $1^{+}$ & $2.96 \pm 0.30 \pm 0.41 \pm 0.48$ & \\
\hline \hline
\end{tabular}

branching fraction is large, it has been separated from the other sources of systematic uncertainty. The $D_{1}(2420)$ resonance decays to $D$ - and $S$-wave states and therefore the two contributions are added; a similar procedure is followed for the $D_{2}(2740)$ resonance, which decays to $P$ - and $F$-wave states.

\section{SUMMARY}

A four-body amplitude analysis of the $B^{-} \rightarrow D^{*+} \pi^{-} \pi^{-}$ decay is performed using $p p$ collision data, corresponding to an integrated luminosity of $4.7 \mathrm{fb}^{-1}$, collected at centerof-mass energies of 7,8 and $13 \mathrm{TeV}$ with the $\mathrm{LHCb}$ detector. Fractional contributions and relative phases for the different resonances contributing in the decay are measured. The data allow for several quasi-model-independent searches for the presence of new states. For the first time, the quantum numbers of previously observed charmed meson resonances are established. In particular the resonance parameters, quantum numbers and partial branching fractions are measured for the $D_{1}(2420), D_{1}(2430)$, $D_{0}(2550), D_{1}^{*}(2600), D_{2}(2740)$ and $D_{3}^{*}(2750)$ resonances. The $J^{P}=1^{+} S$ and $J^{P}=0^{-}$QMI amplitudes give indications for the presence of higher mass $D_{1}$ and $D_{0}^{\prime}$ resonances in the $2.80 \mathrm{GeV}$ mass region. The data are fitted allowing for mixing between $D_{1}$ and $D_{1}^{\prime}$ resonances and their mixing parameters are measured. In particular, the mixing angle deviates from zero by $2.3 \sigma$.

\section{ACKNOWLEDGMENTS}

We express our gratitude to our colleagues in the CERN accelerator departments for the excellent performance of the LHC. We thank the technical and administrative staff at the LHCb institutes. We acknowledge support from CERN and from the national agencies: CAPES, CNPq, FAPERJ and FINEP (Brazil); MOST and NSFC (China); CNRS/IN2P3 (France); BMBF, DFG and MPG (Germany); INFN (Italy); NWO (Netherlands); MNiSW and NCN (Poland); MEN/IFA (Romania); MSHE (Russia); MinECo (Spain); SNSF and SER (Switzerland); NASU (Ukraine); STFC (United Kingdom); DOE NP and NSF (USA). We acknowledge the computing resources that are provided by CERN, IN2P3 (France), KIT and DESY (Germany), INFN (Italy), SURF (Netherlands), PIC (Spain), GridPP (United Kingdom), RRCKI and Yandex LLC (Russia), CSCS (Switzerland), IFIN-HH (Romania), CBPF (Brazil), PL-GRID (Poland) and OSC (USA). We are indebted to the communities behind the multiple open-source software packages on which we depend. Individual groups or members have received support from AvH Foundation (Germany); EPLANET, Marie Skłodowska-Curie Actions and ERC (European Union); ANR, Labex P2IO and OCEVU, and Région AuvergneRhône-Alpes (France); Key Research Program of Frontier Sciences of CAS, CAS PIFI, and the Thousand Talents Program (China); RFBR, RSF and Yandex LLC (Russia); GVA, XuntaGal and GENCAT (Spain); the Royal Society and the Leverhulme Trust (United Kingdom).
[1] S. Godfrey and N. Isgur, Mesons in a relativized quark model with chromodynamics, Phys. Rev. D 32, 189 (1985).

[2] S. Godfrey and K. Moats, Properties of excited charm and charm-strange mesons, Phys. Rev. D 93, 034035 (2016).
[3] M. Tanabashi et al. (Particle Data Group), Review of particle physics, Phys. Rev. D 98, 030001 (2018).

[4] K. Abe et al. (Belle Collaboration), Study of $B^{-} \rightarrow D^{* * 0} \pi^{-}$ $\left(D^{* * 0} \rightarrow D^{(*)+} \pi^{-}\right)$decays, Phys. Rev. D 69, 112002 (2004). 
[5] B. Aubert et al. (BABAR Collaboration), Dalitz plot analysis of $B^{-} \rightarrow D^{+} \pi^{-} \pi^{-}$, Phys. Rev. D 79, 112004 (2009).

[6] R. Aaij et al. (LHCb Collaboration), First observation and amplitude analysis of the $B^{-} \rightarrow D^{+} K^{-} \pi^{-}$decay, Phys. Rev. D 91, 092002 (2015); Erratum, Phys. Rev. D 93, 119901 (2016).

[7] R. Aaij et al. (LHCb Collaboration), Dalitz plot analysis of $B^{0} \rightarrow \bar{D}^{0} \pi^{+} \pi^{-}$decays, Phys. Rev. D 92, 032002 (2015).

[8] R. Aaij et al. (LHCb Collaboration), Amplitude analysis of $B^{-} \rightarrow D^{+} \pi^{-} \pi^{-}$decays, Phys. Rev. D 94, 072001 (2016).

[9] B. Aubert et al. (BABAR Collaboration), Measurement of the branching fractions of $\bar{B} \rightarrow D^{* *} \ell^{-} \bar{\nu}_{\ell}$ decays in events tagged by a fully reconstructed B meson, Phys. Rev. Lett. 101, 261802 (2008).

[10] D. Liventsev et al. (Belle Collaboration), Study of $B \rightarrow$ $D^{* *} l \nu$ with full reconstruction tagging, Phys. Rev. D 77, 091503 (2008).

[11] P. del Amo Sanchez et al. (BABAR Collaboration), Observation of new resonances decaying to $D \pi$ and $D^{*} \pi$ in inclusive $e^{+} e^{-}$collisions near $\sqrt{s}=10.58 \mathrm{GeV}$, Phys. Rev. D 82, 111101 (2010).

[12] R. Aaij et al. (LHCb Collaboration), Study of $D_{J}$ meson decays to $D^{+} \pi^{-}, D^{0} \pi^{+}$and $D^{*+} \pi^{-}$final states in pp collision, J. High Energy Phys. 09 (2013) 145.

[13] R. Aaij et al. (LHCb Collaboration), Dalitz plot analysis of $B^{0} \rightarrow \bar{D}^{0} \pi^{+} \pi^{-}$decays, Phys. Rev. D 92, 032002 (2015).

[14] R. Aaij et al. (LHCb Collaboration), Amplitude analysis of $B^{-} \rightarrow D^{+} \pi^{-} \pi^{-}$decays, Phys. Rev. D 94, 072001 (2016).

[15] A. A. Alves Jr. et al. (LHCb Collaboration), The LHCb detector at the LHC, J. Instrum. 3, S08005 (2008).

[16] R. Aaij et al. (LHCb Collaboration), LHCb detector performance, Int. J. Mod. Phys. A 30, 1530022 (2015).

[17] V. V. Gligorov and M. Williams, Efficient, reliable and fast high-level triggering using a bonsai boosted decision tree, J. Instrum. 8, P02013 (2013).

[18] T. Sjöstrand, S. Mrenna, and P. Skands, PYTHIA 6.4 Physics and manual, J. High Energy Phys. 05 (2006) 026.

[19] I. Belyaev et al., Handling of the generation of primary events in Gauss, the LHCb simulation framework, Nuclear Science Symposium Conference Record (NSS/MIC) (IEEE, 2010), p. 1155.

[20] D. J. Lange, The EvtGen particle decay simulation package, Nucl. Instrum. Methods Phys. Res., Sect. A 462, 152 (2001).
[21] P. Golonka and Z. Was, PHOTOS Monte Carlo: A precision tool for QED corrections in $Z$ and $W$ decays, Eur. Phys. J. C 45, 97 (2006).

[22] J. Allison et al. (GEANT4 Collaboration), Geant4 developments and applications, IEEE Trans. Nucl. Sci. 53, 270 (2006); S. Agostinelli et al. (GEANT4 Collaboration), Geant4: A simulation toolkit, Nucl. Instrum. Methods Phys. Res., Sect. A 506, 250 (2003).

[23] M. Clemencic, G. Corti, S. Easo, C. R. Jones, S. Miglioranzi, M. Pappagallo, and P. Robbe, The LHCb simulation application, Gauss: Design, evolution and experience, J. Phys. Conf. Ser. 331, 032023 (2011).

[24] R. Aaij et al. (LHCb Collaboration), Measurements of the $\Lambda_{b}^{0}, \Xi_{b}^{-}$, and $\Omega_{b}^{-}$Baryon Masses, Phys. Rev. Lett. 110, 182001 (2013).

[25] R. Aaij et al. (LHCb Collaboration), Precision measurement of $D$ meson mass differences, J. High Energy Phys. 06 (2013) 065.

[26] R. Aaij et al. (LHCb Collaboration), Observation of the $B^{+} \rightarrow D^{*-} K^{+} \pi^{+}$decay, Phys. Rev. D 96, 011101 (2017).

[27] C. Zemach, Three pion decays of unstable particles, Phys. Rev. 133, B1201 (1964).

[28] C. Dionisi et al. (CERN-Collège de France-Madrid-Stockholm Collaboration), Observation and quantum numbers determination of the $\mathrm{E}(1420)$ meson in $\pi^{-} p$ interactions at 3.95-GeV/c, Nucl. Phys. B169, 1 (1980).

[29] V. Filippini, A. Fontana, and A. Rotondi, Covariant spin tensors in meson spectroscopy, Phys. Rev. D 51, 2247 (1995).

[30] J. M. Blatt and V. F. Weisskopf, Theoretical Nuclear Physics (John Wiley \& Sons, New York, 1952).

[31] E. M. Aitala et al. (E791 Collaboration), Model independent measurement of S-wave $K^{-} \pi^{+}$systems using $D^{+} \rightarrow K^{-} \pi^{+} \pi^{+}$decays from Fermilab E791, Phys. Rev. D 73, 032004 (2006); Erratum, Phys. Rev. D 74, 059901 (2006).

[32] J. P. Lees et al. (BABAR Collaboration), Measurement of the $\mathrm{I}=1 / 2 K \pi \mathcal{S}$-wave amplitude from Dalitz plot analyses of $\eta_{c} \rightarrow K \bar{K} \pi$ in two-photon interactions, Phys. Rev. D 93, 012005 (2016).

[33] N. Isgur and M. B. Wise, Spectroscopy with Heavy Quark Symmetry, Phys. Rev. Lett. 66, 1130 (1991).

R. Aaij, ${ }^{30}$ C. Abellán Beteta, ${ }^{47}$ T. Ackernley,${ }^{57}$ B. Adeva, ${ }^{44}$ M. Adinolfi, ${ }^{51}$ H. Afsharnia, ${ }^{8}$ C. A. Aidala, ${ }^{78}$ S. Aiola, ${ }^{24}$ Z. Ajaltouni, ${ }^{8}$ S. Akar, ${ }^{62}$ P. Albicocco, ${ }^{21}$ J. Albrecht, ${ }^{13}$ F. Alessio, ${ }^{45}$ M. Alexander, ${ }^{56}$ A. Alfonso Albero, ${ }^{43}$ G. Alkhazov, ${ }^{36}$ P. Alvarez Cartelle, ${ }^{58}$ A. A. Alves Jr., ${ }^{44}$ S. Amato, ${ }^{2}$ Y. Amhis, ${ }^{10}$ L. An, ${ }^{20}$ L. Anderlini, ${ }^{20}$ G. Andreassi, ${ }^{46}$ M. Andreotti, ${ }^{19}$ F. Archilli, ${ }^{15}$ J. Arnau Romeu, ${ }^{9}$ A. Artamonov, ${ }^{42}$ M. Artuso, ${ }^{65}$ K. Arzymatov, ${ }^{40}$ E. Aslanides, ${ }^{9}$ M. Atzeni,${ }^{47}$ B. Audurier, ${ }^{25}$ S. Bachmann, ${ }^{15}$ J. J. Back ${ }^{53}$ S. Baker,${ }^{58}$ V. Balagura, ${ }^{10, b}$ W. Baldini, ${ }^{19,45}$ A. Baranov, ${ }^{40}$ R. J. Barlow, ${ }^{59}$ S. Barsuk,${ }^{10}$ W. Barter ${ }^{58}$ M. Bartolini, ${ }^{22, c}$ F. Baryshnikov, ${ }^{74}$ G. Bassi,${ }^{27}$ V. Batozskaya, ${ }^{34}$ B. Batsukh, ${ }^{65}$ A. Battig, ${ }^{13}$ V. Battista, ${ }^{46}$ A. Bay, ${ }^{46}$ M. Becker,${ }^{13}$ F. Bedeschi, ${ }^{27}$ I. Bediaga, ${ }^{1}$ A. Beiter, ${ }^{65}$ L. J. Bel,${ }^{30}$ V. Belavin, ${ }^{40}$ S. Belin, ${ }^{25}$ N. Beliy, ${ }^{4}$ V. Bellee,${ }^{46}$ K. Belous, ${ }^{42}$ I. Belyaev, ${ }^{37}$ G. Bencivenni, ${ }^{21}$ E. Ben-Haim, ${ }^{11}$ S. Benson, ${ }^{30}$ S. Beranek, ${ }^{12}$ A. Berezhnoy,${ }^{38}$ R. Bernet, ${ }^{47}$ D. Berninghoff, ${ }^{15}$ H. C. Bernstein, ${ }^{65}$ E. Bertholet, ${ }^{11}$ A. Bertolin, ${ }^{26}$ C. Betancourt,${ }^{47}$ F. Betti, ${ }^{18, d}$ M. O. Bettler, ${ }^{52}$ Ia. Bezshyiko, ${ }^{47}$ S. Bhasin, ${ }^{51}$ J. Bhom, ${ }^{32}$ M. S. Bieker, ${ }^{13}$ S. Bifani, ${ }^{50}$ P. Billoir, ${ }^{11}$ A. Birnkraut, ${ }^{13}$ A. Bizzeti, ${ }^{20, \mathrm{e}}$ M. Bjørn, ${ }^{60}$ 
M. P. Blago, ${ }^{45}$ T. Blake, ${ }^{53}$ F. Blanc, ${ }^{46}$ S. Blusk, ${ }^{65}$ D. Bobulska, ${ }^{56}$ V. Bocci, ${ }^{29}$ O. Boente Garcia, ${ }^{44}$ T. Boettcher, ${ }^{61}$ A. Boldyrev, ${ }^{75}$ A. Bondar, ${ }^{41, f}$ N. Bondar, ${ }^{36}$ S. Borghi, ${ }^{59,45}$ M. Borisyak, ${ }^{40}$ M. Borsato, ${ }^{15}$ J. T. Borsuk, ${ }^{32}$ M. Boubdir, ${ }^{12}$ T. J. V. Bowcock,${ }^{57}$ C. Bozzi, ${ }^{19,45}$ S. Braun, ${ }^{15}$ A. Brea Rodriguez, ${ }^{44}$ M. Brodski, ${ }^{45}$ J. Brodzicka, ${ }^{32}$ A. Brossa Gonzalo,${ }^{53}$ D. Brundu, ${ }^{25,45}$ E. Buchanan, ${ }^{51}$ A. Buonaura, ${ }^{47}$ C. Burr, ${ }^{45}$ A. Bursche, ${ }^{25}$ J. S. Butter, ${ }^{30}$ J. Buytaert, ${ }^{45}$ W. Byczynski, ${ }^{45}$ S. Cadeddu, ${ }^{25}$ H. Cai,${ }^{69}$ R. Calabrese, ${ }^{19,9}$ S. Cali, ${ }^{21}$ R. Calladine, ${ }^{50}$ M. Calvi, ${ }^{23, h}$ M. Calvo Gomez, ${ }^{43, i}$ A. Camboni, ${ }^{43, i}$ P. Campana, ${ }^{21}$ D. H. Campora Perez, ${ }^{45}$ L. Capriotti, ${ }^{18, d}$ A. Carbone, ${ }^{18, d}$ G. Carboni,${ }^{28}$ R. Cardinale, ${ }^{22, c}$ A. Cardini, ${ }^{25}$ P. Carniti, ${ }^{23, h}$ K. Carvalho Akiba ${ }^{30}$ A. Casais Vidal,${ }^{44}$ G. Casse,${ }^{57}$ M. Cattaneo, ${ }^{45}$ G. Cavallero, ${ }^{22}$ R. Cenci, ${ }^{27, j}$ J. Cerasoli, ${ }^{9}$ M. G. Chapman, ${ }^{51}$ M. Charles, ${ }^{11,45}$ Ph. Charpentier, ${ }^{45}$ G. Chatzikonstantinidis,${ }^{50}$ M. Chefdeville, ${ }^{7}$ V. Chekalina, ${ }^{40}$ C. Chen, ${ }^{3}$ S. Chen, ${ }^{25}$ A. Chernov, ${ }^{32}$ S.-G. Chitic, ${ }^{45}$ V. Chobanova, ${ }^{44}$ M. Chrzaszcz, ${ }^{45}$ A. Chubykin, ${ }^{36}$ P. Ciambrone,${ }^{21}$ M. F. Cicala, ${ }^{53}$ X. Cid Vidal, ${ }^{44}$ G. Ciezarek, ${ }^{45}$ F. Cindolo, ${ }^{18}$ P. E. L. Clarke, ${ }^{55}$ M. Clemencic, ${ }^{45}$ H. V. Cliff, ${ }^{52}$ J. Closier, ${ }^{45}$ J. L. Cobbledick, ${ }^{59}$ V. Coco, ${ }^{45}$ J. A. B. Coelho, ${ }^{10}$ J. Cogan, ${ }^{9}$ E. Cogneras, ${ }^{8}$ L. Cojocariu, ${ }^{35}$ P. Collins,${ }^{45}$ T. Colombo, ${ }^{45}$ A. Comerma-Montells, ${ }^{15}$ A. Contu, ${ }^{25}$ N. Cooke, ${ }^{50}$ G. Coombs ${ }^{56}$ S. Coquereau, ${ }^{43}$ G. Corti, ${ }^{45}$ C. M. Costa Sobral, ${ }^{53}$ B. Couturier, ${ }^{45}$ G. A. Cowan, ${ }^{55}$ D. C. Craik, ${ }^{61}$ A. Crocombe ${ }^{53}$ M. Cruz Torres, ${ }^{1}$ R. Currie,${ }^{55}$ C. L. Da Silva,${ }^{64}$ E. Dall'Occo,${ }^{30}$ J. Dalseno, ${ }^{44,51}$ C. D’Ambrosio, ${ }^{45}$ A. Danilina, ${ }^{37}$ P. d'Argent, ${ }^{15}$ A. Davis, ${ }^{59}$ O. De Aguiar Francisco, ${ }^{45}$ K. De Bruyn, ${ }^{45}$ S. De Capua, ${ }^{59}$ M. De Cian, ${ }^{46}$ J. M. De Miranda, ${ }^{1}$ L. De Paula, ${ }^{2}$ M. De Serio,,${ }^{17, k}$ P. De Simone,${ }^{21}$ J. A. de Vries,${ }^{30}$ C. T. Dean,${ }^{64}$ W. Dean,${ }^{78}$ D. Decamp, ${ }^{7}$ L. Del Buono, ${ }^{11}$ B. Delaney ${ }^{52}$ H.-P. Dembinski, ${ }^{14}$ M. Demmer, ${ }^{13}$ A. Dendek ${ }^{33}$ V. Denysenko, ${ }^{47}$ D. Derkach,${ }^{75}$ O. Deschamps ${ }^{8}$ F. Desse,${ }^{10}$ F. Dettori,${ }^{25}$ B. Dey, ${ }^{6}$ A. Di Canto, ${ }^{45}$ P. Di Nezza,${ }^{21}$ S. Didenko, ${ }^{74}$ H. Dijkstra, ${ }^{45}$ F. Dordei,${ }^{25}$ M. Dorigo, ${ }^{27,1}$ A. C. dos Reis, ${ }^{1}$ A. Dosil Suárez, ${ }^{44}$ L. Douglas, ${ }^{56}$ A. Dovbnya, ${ }^{48}$ K. Dreimanis, ${ }^{57}$ M. W. Dudek, ${ }^{32}$ L. Dufour, ${ }^{45}$ G. Dujany, ${ }^{11}$ P. Durante, ${ }^{45}$ J. M. Durham, ${ }^{64}$ D. Dutta, ${ }^{59}$ R. Dzhelyadin, ${ }^{42, a}$ M. Dziewiecki, ${ }^{15}$ A. Dziurda, ${ }^{32}$ A. Dzyuba, ${ }^{36}$ S. Easo, ${ }^{54}$ U. Egede, ${ }^{58}$ V. Egorychev,${ }^{37}$ S. Eidelman, ${ }^{41, f}$ S. Eisenhardt,${ }^{55}$ R. Ekelhof, ${ }^{13}$ S. Ek-In, ${ }^{46}$ L. Eklund, ${ }^{56}$ S. Ely, ${ }^{65}$ A. Ene, ${ }^{35}$ S. Escher, ${ }^{12}$ S. Esen,${ }^{30}$ T. Evans, ${ }^{45}$ A. Falabella, ${ }^{18}$ J. Fan, ${ }^{3}$ N. Farley, ${ }^{50}$ S. Farry, ${ }^{57}$ D. Fazzini, ${ }^{10}$ M. Féo, ${ }^{45}$ P. Fernandez Declara, ${ }^{45}$ A. Fernandez Prieto, ${ }^{44}$ F. Ferrari, ${ }^{18, d}$ L. Ferreira Lopes,${ }^{46}$ F. Ferreira Rodrigues,${ }^{2}$ S. Ferreres Sole, ${ }^{30}$ M. Ferro-Luzzi, ${ }^{45}$ S. Filippov, ${ }^{39}$ R. A. Fini, ${ }^{17}$ M. Fiorini, ${ }^{19,}{ }^{1}$ M. Firlej, ${ }^{33}$ K. M. Fischer, ${ }^{60}$ C. Fitzpatrick, ${ }^{45}$ T. Fiutowski, ${ }^{33}$ F. Fleuret, ${ }^{10, \mathrm{~b}}$ M. Fontana ${ }^{45}$ F. Fontanelli, ${ }^{22, \mathrm{c}}$ R. Forty, ${ }^{45}$ V. Franco Lima,${ }^{57}$ M. Franco Sevilla,${ }^{63}$ M. Frank,${ }^{45}$ C. Frei, ${ }^{45}$ D. A. Friday, ${ }^{56}$ J. Fu, ${ }^{24, \mathrm{~m}}$ W. Funk,${ }^{45}$ E. Gabriel,${ }^{55}$ A. Gallas Torreira, ${ }^{44}$ D. Galli, ${ }^{18, \mathrm{~d}}$ S. Gallorini, ${ }^{26}$ S. Gambetta, ${ }^{55}$ Y. Gan, ${ }^{3}$ M. Gandelman, ${ }^{2}$ P. Gandini, ${ }^{24}$ Y. Gao, ${ }^{3}$ L. M. Garcia Martin, ${ }^{77}$ J. García Pardiñas, ${ }^{47}$ B. Garcia Plana,,${ }^{44}$

F. A. Garcia Rosales ${ }^{10}$ J. Garra Tico,${ }^{52}$ L. Garrido, ${ }^{43}$ D. Gascon,${ }^{43}$ C. Gaspar,${ }^{45}$ G. Gazzoni, ${ }^{8}$ D. Gerick,${ }^{15}$ E. Gersabeck, ${ }^{59}$ M. Gersabeck, ${ }^{59}$ T. Gershon, ${ }^{53}$ D. Gerstel, ${ }^{9}$ Ph. Ghez, ${ }^{7}$ V. Gibson, ${ }^{52}$ A. Gioventù,${ }^{44}$ O. G. Girard,${ }^{46}$ P. Gironella Gironell, ${ }^{43}$ L. Giubega, ${ }^{35}$ C. Giugliano, ${ }^{19}$ K. Gizdov, ${ }^{55}$ V. V. Gligorov, ${ }^{11}$ C. Göbel,${ }^{67}$ D. Golubkov, ${ }^{37}$ A. Golutvin, ${ }^{58,74}$ A. Gomes, ${ }^{1, n}$ I. V. Gorelov, ${ }^{38}$ C. Gotti, ${ }^{23, h}$ E. Govorkova, ${ }^{30}$ J. P. Grabowski, ${ }^{15}$ R. Graciani Diaz, ${ }^{43}$ T. Grammatico, ${ }^{11}$

L. A. Granado Cardoso, ${ }^{45}$ E. Graugés, ${ }^{43}$ E. Graverini, ${ }^{46}$ G. Graziani, ${ }^{20}$ A. Grecu, ${ }^{35}$ R. Greim, ${ }^{30}$ P. Griffith, ${ }^{19}$ L. Grillo, ${ }^{59}$ L. Gruber, ${ }^{45}$ B. R. Gruberg Cazon, ${ }^{60}$ C. Gu, ${ }^{3}$ E. Gushchin, ${ }^{39}$ A. Guth, ${ }^{12}$ Yu. Guz, ${ }^{42,45}$ T. Gys, ${ }^{45}$ T. Hadavizadeh, ${ }^{60}$ C. Hadjivasiliou, ${ }^{8}$ G. Haefeli, ${ }^{46}$ C. Haen, ${ }^{45}$ S. C. Haines,${ }^{52}$ P. M. Hamilton, ${ }^{63}$ Q. Han,${ }^{6}$ X. Han, ${ }^{15}$ T. H. Hancock, ${ }^{60}$ S. Hansmann-Menzemer, ${ }^{15}$ N. Harnew, ${ }^{60}$ T. Harrison, ${ }^{57}$ C. Hasse, ${ }^{45}$ M. Hatch,${ }^{45}$ J. He, ${ }^{4}$ M. Hecker, ${ }^{58}$ K. Heijhoff, ${ }^{30}$ K. Heinicke, ${ }^{13}$ A. Heister, ${ }^{13}$ A. M. Hennequin, ${ }^{45}$ K. Hennessy, ${ }^{57}$ L. Henry, ${ }^{77}$ M. Heß,${ }^{71}$ J. Heuel, ${ }^{12}$ A. Hicheur, ${ }^{66}$ R. Hidalgo Charman, ${ }^{59}$ D. Hill, ${ }^{60}$ M. Hilton, ${ }^{59}$ P. H. Hopchev, ${ }^{46}$ J. Hu, ${ }^{15}$ W. Hu, ${ }^{6}$ W. Huang, ${ }^{4}$ Z. C. Huard, ${ }^{62}$ W. Hulsbergen, ${ }^{30}$ T. Humair, ${ }^{58}$ R. J. Hunter, ${ }^{53}$ M. Hushchyn, ${ }^{75}$ D. Hutchcroft, ${ }^{57}$ D. Hynds, ${ }^{30}$ P. Ibis, ${ }^{13}$ M. Idzik, ${ }^{33}$ P. Ilten,,${ }^{50}$ A. Inglessi ${ }^{36}$ A. Inyakin, ${ }^{42}$ K. Ivshin, ${ }^{36}$ R. Jacobsson, ${ }^{45}$ S. Jakobsen, ${ }^{45}$ J. Jalocha, ${ }^{60}$ E. Jans, ${ }^{30}$ B. K. Jashal, ${ }^{77}$ A. Jawahery, ${ }^{63}$ V. Jevtic, ${ }^{13}$ F. Jiang, ${ }^{3}$ M. John, ${ }^{60}$ D. Johnson, ${ }^{45}$ C. R. Jones, ${ }^{52}$ B. Jost,${ }^{45}$ N. Jurik, ${ }^{60}$ S. Kandybei, ${ }^{48}$ M. Karacson, ${ }^{45}$ J. M. Kariuki, ${ }^{51}$ S. Karodia,${ }^{56}$ N. Kazeev, ${ }^{75}$ M. Kecke, ${ }^{15}$ F. Keizer,${ }^{52}$ M. Kelsey, ${ }^{65}$ M. Kenzie, ${ }^{52}$ T. Ketel, ${ }^{31}$ B. Khanji ${ }^{45}$ A. Kharisova, ${ }^{76}$ C. Khurewathanakul, ${ }^{46}$ K. E. Kim,${ }^{65}$ T. Kirn, ${ }^{12}$ V. S. Kirsebom, ${ }^{46}$ S. Klaver, ${ }^{21}$ K. Klimaszewski, ${ }^{34}$ S. Koliiev, ${ }^{49}$ A. Kondybayeva, ${ }^{74}$ A. Konoplyannikov, ${ }^{37}$ P. Kopciewicz, ${ }^{33}$ R. Kopecna, ${ }^{15}$ P. Koppenburg, ${ }^{30}$ I. Kostiuk, ${ }^{30,49}$ O. Kot, ${ }^{49}$ S. Kotriakhova, ${ }^{36}$ M. Kozeiha, ${ }^{8}$ L. Kravchuk ${ }^{39}$ R. D. Krawczyk, ${ }^{45}$ M. Kreps,${ }^{53}$ F. Kress, ${ }^{58}$ S. Kretzschmar, ${ }^{12}$ P. Krokovny, ${ }^{41, \mathrm{f}}$ W. Krupa ${ }^{33}$ W. Krzemien, ${ }^{34}$ W. Kucewicz, ${ }^{32,0}$ M. Kucharczyk, ${ }^{32}$ V. Kudryavtsev, ${ }^{41, f}$ H. S. Kuindersma, ${ }^{30}$ G. J. Kunde, ${ }^{64}$ A. K. Kuonen, ${ }^{46}$ T. Kvaratskheliya, ${ }^{37}$ D. Lacarrere, ${ }^{45}$ G. Lafferty, ${ }^{59}$ A. Lai, ${ }^{25}$ D. Lancierini, ${ }^{47}$ J. J. Lane,${ }^{59}$ G. Lanfranchi, ${ }^{21}$ C. Langenbruch, ${ }^{12}$ T. Latham, ${ }^{53}$ F. Lazzari, ${ }^{27, p}$ C. Lazzeroni, ${ }^{50}$ R. Le Gac, ${ }^{9}$ R. Lefèvre, ${ }^{8}$ A. Leflat, ${ }^{38}$ F. Lemaitre, ${ }^{45}$ O. Leroy, ${ }^{9}$ T. Lesiak, ${ }^{32}$ B. Leverington, ${ }^{15} \mathrm{H} . \mathrm{Li},{ }^{68}$ P.-R. Li, ${ }^{4, \mathrm{q}} \mathrm{X} . \mathrm{Li},{ }^{64} \mathrm{Y} . \mathrm{Li},{ }^{5} \mathrm{Z} . \mathrm{Li},{ }^{65} \mathrm{X}$. Liang, ${ }^{65}$ R. Lindner, ${ }^{45}$ F. Lionetto, ${ }^{47}$ V. Lisovskyi, ${ }^{10}$ G. Liu, ${ }^{68}$ X. Liu, ${ }^{3}$ D. Loh, ${ }^{53}$ A. Loi, ${ }^{25}$ J. Lomba Castro, ${ }^{44}$ I. Longstaff, ${ }^{56}$ J. H. Lopes, ${ }^{2}$ G. Loustau, ${ }^{47}$ G. H. Lovell, ${ }^{52}$ D. Lucchesi, ${ }^{26, \mathrm{r}}$ M. Lucio Martinez, ${ }^{30}$ Y. Luo, ${ }^{3}$ A. Lupato, ${ }^{26}$ E. Luppi, ${ }^{19, \mathrm{~g}}$ O. Lupton, ${ }^{53}$ 
A. Lusiani, ${ }^{27, \mathrm{~s}}$ X. Lyu ${ }^{4}$ S. Maccolini,${ }^{18, \mathrm{~d}}$ F. Machefert, ${ }^{10}$ F. Maciuc, ${ }^{35}$ V. Macko, ${ }^{46}$ P. Mackowiak, ${ }^{13}$ S. Maddrell-Mander,${ }^{51}$ L. R. Madhan Mohan, ${ }^{51}$ O. Maev, ${ }^{36,45}$ A. Maevskiy, ${ }^{75}$ K. Maguire, ${ }^{59}$ D. Maisuzenko, ${ }^{36}$ M. W. Majewski, ${ }^{33}$ S. Malde, ${ }^{60}$ B. Malecki, ${ }^{45}$ A. Malinin, ${ }^{73}$ T. Maltsev ${ }^{41, \mathrm{f}}$ H. Malygina, ${ }^{15}$ G. Manca, ${ }^{25, \mathrm{t}}$ G. Mancinelli, ${ }^{9}$ D. Manuzzi, ${ }^{18, \mathrm{~d}}$ D. Marangotto, ${ }^{24, \mathrm{~m}}$ J. Maratas, ${ }^{8, u}$ J. F. Marchand, ${ }^{7}$ U. Marconi, ${ }^{18}$ S. Mariani, ${ }^{20}$ C. Marin Benito, ${ }^{10}$ M. Marinangeli, ${ }^{46}$ P. Marino, ${ }^{46}$ J. Marks, ${ }^{15}$ P. J. Marshall, ${ }^{57}$ G. Martellotti, ${ }^{29}$ L. Martinazzoli, ${ }^{45}$ M. Martinelli, ${ }^{45,23, \mathrm{~h}}$ D. Martinez Santos, ${ }^{44}$ F. Martinez Vidal, ${ }^{77}$

A. Massafferri, ${ }^{1}$ M. Materok, ${ }^{12}$ R. Matev, ${ }^{45}$ A. Mathad, ${ }^{47}$ Z. Mathe, ${ }^{45}$ V. Matiunin, ${ }^{37}$ C. Matteuzzi, ${ }^{23}$ K. R. Mattioli, ${ }^{78}$ A. Mauri, ${ }^{47}$ E. Maurice, ${ }^{10, b}$ M. McCann,${ }^{58,45}$ L. Mcconnell, ${ }^{16}$ A. McNab,${ }^{59}$ R. McNulty, ${ }^{16}$ J. V. Mead,${ }^{57}$ B. Meadows,${ }^{62}$ C. Meaux,${ }^{9}$ N. Meinert, ${ }^{71}$ D. Melnychuk,${ }^{34}$ S. Meloni, ${ }^{23, \mathrm{~h}}$ M. Merk, ${ }^{30}$ A. Merli, ${ }^{24, \mathrm{~m}}$ E. Michielin, ${ }^{26}$ D. A. Milanes, ${ }^{70}$ E. Millard, ${ }^{53}$ M.-N. Minard, ${ }^{7}$ O. Mineev, ${ }^{37}$ L. Minzoni, ${ }^{19, \mathrm{~g}}$ S. E. Mitchell,${ }^{55}$ B. Mitreska, ${ }^{59}$ D. S. Mitzel, ${ }^{45}$ A. Mödden, ${ }^{13}$ A. Mogini, ${ }^{11}$ R. D. Moise, ${ }^{58}$ T. Mombächer, ${ }^{13}$ I. A. Monroy, ${ }^{70}$ S. Monteil, ${ }^{8}$ M. Morandin, ${ }^{26}$ G. Morello, ${ }^{21}$ M. J. Morello,,${ }^{27, s}$ J. Moron, ${ }^{33}$ A. B. Morris, ${ }^{9}$ A. G. Morris, ${ }^{53}$ R. Mountain, ${ }^{65}$ H. Mu, ${ }^{3}$ F. Muheim,${ }^{55}$ M. Mukherjee, ${ }^{6}$ M. Mulder, ${ }^{30}$ D. Müller, ${ }^{45}$ J. Müller, ${ }^{13}$ K. Müller, ${ }^{47}$ V. Müller, ${ }^{13}$ C. H. Murphy, ${ }^{60}$ D. Murray,${ }^{59}$ P. Muzzetto,${ }^{25}$ P. Naik, ${ }^{51}$ T. Nakada, ${ }^{46}$ R. Nandakumar, ${ }^{54}$

A. Nandi, ${ }^{60}$ T. Nanut,${ }^{46}$ I. Nasteva, ${ }^{2}$ M. Needham, ${ }^{55}$ N. Neri, ${ }^{24, m}$ S. Neubert, ${ }^{15}$ N. Neufeld,${ }^{45}$ R. Newcombe,${ }^{58}$

T. D. Nguyen, ${ }^{46}$ C. Nguyen-Mau, ${ }^{46, v}$ E. M. Niel, ${ }^{10}$ S. Nieswand, ${ }^{12}$ N. Nikitin, ${ }^{38}$ N. S. Nolte, ${ }^{45}$ A. Oblakowska-Mucha, ${ }^{33}$ V. Obraztsov, ${ }^{42}$ S. Ogilvy, ${ }^{56}$ D. P. O'Hanlon, ${ }^{18}$ R. Oldeman, ${ }^{25, t}$ C. J. G. Onderwater, ${ }^{72}$ J. D. Osborn, ${ }^{78}$ A. Ossowska, ${ }^{32}$ J. M. Otalora Goicochea, ${ }^{2}$ T. Ovsiannikova, ${ }^{37}$ P. Owen, ${ }^{47}$ A. Oyanguren, ${ }^{77}$ P. R. Pais, ${ }^{46}$ T. Pajero, ${ }^{27, s}$ A. Palano, ${ }^{17, \dagger}$ M. Palutan, ${ }^{21}$ G. Panshin, ${ }^{76}$ A. Papanestis, ${ }^{54}$ M. Pappagallo, ${ }^{55}$ L. L. Pappalardo, ${ }^{19, g}$ W. Parker, ${ }^{63}$ C. Parkes,${ }^{59,45}$ G. Passaleva, ${ }^{20,45}$ A. Pastore, ${ }^{17}$ M. Patel,${ }^{58}$ C. Patrignani, ${ }^{18, d}$ A. Pearce, ${ }^{45}$ A. Pellegrino, ${ }^{30}$ G. Penso, ${ }^{29}$ M. Pepe Altarelli, ${ }^{45}$ S. Perazzini ${ }^{18}$ D. Pereima,${ }^{37}$ P. Perret,${ }^{8}$ L. Pescatore,${ }^{46}$ K. Petridis,${ }^{51}$ A. Petrolini, ${ }^{22, c}$ A. Petrov, ${ }^{73}$ S. Petrucci, ${ }^{55}$ M. Petruzzo, ${ }^{24, \mathrm{~m}}$ B. Pietrzyk, ${ }^{7}$ G. Pietrzyk, ${ }^{46}$ M. Pikies,${ }^{32}$ M. Pili, ${ }^{60}$ D. Pinci, ${ }^{29}$ J. Pinzino, ${ }^{45}$ F. Pisani, ${ }^{45}$ A. Piucci, ${ }^{15}$ V. Placinta, ${ }^{35}$ S. Playfer, ${ }^{55}$ J. Plews, ${ }^{50}$ M. Plo Casasus, ${ }^{44}$ F. Polci, ${ }^{11}$ M. Poli Lener, ${ }^{21}$ M. Poliakova, ${ }^{65}$ A. Poluektov, ${ }^{9}$ N. Polukhina, ${ }^{74, w}$ I. Polyakov, ${ }^{65}$ E. Polycarpo, ${ }^{2}$ G. J. Pomery,${ }^{51}$ S. Ponce,${ }^{45}$ A. Popov, ${ }^{42}$ D. Popov,${ }^{50}$ S. Poslavskii, ${ }^{42}$ K. Prasanth, ${ }^{32}$ L. Promberger, ${ }^{45}$ C. Prouve, ${ }^{44}$ V. Pugatch, ${ }^{49}$ A. Puig Navarro, ${ }^{47}$ H. Pullen, ${ }^{60}$ G. Punzi, ${ }^{27, j}$ W. Qian, ${ }^{4}$ J. Qin, ${ }^{4}$ R. Quagliani, ${ }^{11}$ B. Quintana, ${ }^{8}$ N. V. Raab,${ }^{16}$ B. Rachwal, ${ }^{33}$ J. H. Rademacker, ${ }^{51}$ M. Rama,${ }^{27}$ M. Ramos Pernas, ${ }^{44}$ M. S. Rangel, ${ }^{2}$ F. Ratnikov, ${ }^{40,75}$ G. Raven, ${ }^{31}$ M. Ravonel Salzgeber, ${ }^{45}$ M. Reboud, ${ }^{7}$ F. Redi, ${ }^{46}$ S. Reichert, ${ }^{13}$ F. Reiss, ${ }^{11}$ C. Remon Alepuz, ${ }^{77}$ Z. Ren, ${ }^{3}$ V. Renaudin, ${ }^{60}$ S. Ricciardi, ${ }^{54}$ S. Richards,${ }^{51}$ K. Rinnert,${ }^{57}$ P. Robbe, ${ }^{10}$ A. Robert, ${ }^{11}$ A. B. Rodrigues, ${ }^{46}$ E. Rodrigues, ${ }^{62}$ J. A. Rodriguez Lopez,${ }^{70}$ M. Roehrken, ${ }^{45}$ S. Roiser ${ }^{45}$ A. Rollings,${ }^{60}$ V. Romanovskiy, ${ }^{42}$ M. Romero Lamas, ${ }^{44}$ A. Romero Vidal, ${ }^{44}$ J. D. Roth, ${ }^{78}$ M. Rotondo, ${ }^{21}$ M. S. Rudolph, ${ }^{65}$ T. Ruf, ${ }^{45}$ J. Ruiz Vidal, ${ }^{77}$ J. Ryzka, ${ }^{33}$ J. J. Saborido Silva, ${ }^{44}$ N. Sagidova, ${ }^{36}$ B. Saitta, ${ }^{25, t}$ C. Sanchez Gras,${ }^{30}$ C. Sanchez Mayordomo, ${ }^{77}$ B. Sanmartin Sedes, ${ }^{44}$ R. Santacesaria, ${ }^{29}$ C. Santamarina Rios, ${ }^{44}$ M. Santimaria, ${ }^{21,45}$ E. Santovetti, ${ }^{28, x}$ G. Sarpis, ${ }^{59}$ A. Sarti, ${ }^{29}$ C. Satriano, ${ }^{29, y}$ A. Satta, ${ }^{28}$ M. Saur, ${ }^{4}$ D. Savrina, ${ }^{37,38}$ L. G. Scantlebury Smead, ${ }^{60}$ S. Schael, ${ }^{12}$ M. Schellenberg, ${ }^{13}$ M. Schiller, ${ }^{56}$ H. Schindler, ${ }^{45}$ M. Schmelling, ${ }^{14}$ T. Schmelzer, ${ }^{13}$ B. Schmidt, ${ }^{45}$ O. Schneider, ${ }^{46}$ A. Schopper, ${ }^{45}$ H. F. Schreiner, ${ }^{62}$ M. Schubiger, ${ }^{30}$ S. Schulte, ${ }^{46}$ M. H. Schune, ${ }^{10}$ R. Schwemmer, ${ }^{45}$ B. Sciascia, ${ }^{21}$ A. Sciubba, ${ }^{29, z}$ S. Sellam, ${ }^{66}$ A. Semennikov, ${ }^{37}$ A. Sergi, ${ }^{50,45}$ N. Serra, ${ }^{47}$ J. Serrano, ${ }^{9}$ L. Sestini, ${ }^{26}$ A. Seuthe, ${ }^{13}$ P. Seyfert, ${ }^{45}$ D. M. Shangase ${ }^{78}$ M. Shapkin, ${ }^{42}$ T. Shears, ${ }^{57}$ L. Shekhtman, ${ }^{41, f}$ V. Shevchenko, ${ }^{73,74}$ E. Shmanin,${ }^{74}$ J. D. Shupperd, ${ }^{65}$ B. G. Siddi, ${ }^{19}$ R. Silva Coutinho, ${ }^{47}$ L. Silva de Oliveira, ${ }^{2}$ G. Simi,${ }^{26, \mathrm{r}}$ S. Simone, ${ }^{17, \mathrm{k}}$ I. Skiba, ${ }^{19}$ N. Skidmore, ${ }^{15}$ T. Skwarnicki, ${ }^{65}$ M. W. Slater, ${ }^{50}$ J. G. Smeaton, ${ }^{52}$ E. Smith, ${ }^{12}$ I. T. Smith,${ }^{55}$ M. Smith, ${ }^{58}$ M. Soares,${ }^{18}$ L. Soares Lavra, ${ }^{1}$ M. D. Sokoloff, ${ }^{62}$ F. J. P. Soler, ${ }^{56}$ B. Souza De Paula, ${ }^{2}$ B. Spaan, ${ }^{13}$ E. Spadaro Norella, ${ }^{24, \mathrm{~m}}$ P. Spradlin, ${ }^{56}$ F. Stagni, ${ }^{45}$ M. Stahl, ${ }^{62}$ S. Stahl, ${ }^{45}$ P. Stefko, ${ }^{46}$ S. Stefkova, ${ }^{58}$ O. Steinkamp, ${ }^{47}$ S. Stemmle, ${ }^{15}$ O. Stenyakin, ${ }^{42}$ M. Stepanova, ${ }^{36}$ H. Stevens, ${ }^{13}$ S. Stone, ${ }^{65}$ S. Stracka, ${ }^{27}$ M. E. Stramaglia, ${ }^{46}$ M. Straticiuc, ${ }^{35}$ U. Straumann, ${ }^{47}$ S. Strokov, ${ }^{76}$ J. Sun, ${ }^{3}$ L. Sun, ${ }^{69}$ Y. Sun, ${ }^{63}$ P. Svihra, ${ }^{59}$ K. Swientek, ${ }^{33}$ A. Szabelski, ${ }^{34}$ T. Szumlak, ${ }^{33}$ M. Szymanski, ${ }^{4}$ S. Taneja, ${ }^{59}$ Z. Tang, ${ }^{3}$ T. Tekampe, ${ }^{13}$ G. Tellarini, ${ }^{19}$ F. Teubert, ${ }^{45}$ E. Thomas, ${ }^{45}$ K. A. Thomson, ${ }^{57}$ M. J. Tilley, ${ }^{58}$ V. Tisserand, ${ }^{8}$ S. T'Jampens, ${ }^{7}$ M. Tobin, ${ }^{5}$ S. Tolk, ${ }^{45}$ L. Tomassetti, ${ }^{19, \mathrm{~g}}$ D. Tonelli, ${ }^{27}$ D. Y. Tou, ${ }^{11}$ E. Tournefier, ${ }^{7}$ M. Traill, ${ }^{56}$ M. T. Tran ${ }^{46}$ A. Trisovic, ${ }^{52}$ A. Tsaregorodtsev, ${ }^{9}$ G. Tuci, ${ }^{27,45, j}$ A. Tully, ${ }^{52}$ N. Tuning, ${ }^{30}$ A. Ukleja,${ }^{34}$ A. Usachov, ${ }^{10}$ A. Ustyuzhanin, ${ }^{40,75}$ U. Uwer, ${ }^{15}$ A. Vagner, ${ }^{76}$ V. Vagnoni, ${ }^{18}$ A. Valassi, ${ }^{45}$ S. Valat,${ }^{45}$ G. Valenti, ${ }^{18}$ M. van Beuzekom, ${ }^{30}$ H. Van Hecke, ${ }^{64}$ E. van Herwijnen, ${ }^{45}$ C. B. Van Hulse, ${ }^{16}$ J. van Tilburg, ${ }^{30}$ M. van Veghel, ${ }^{72}$ R. Vazquez Gomez, ${ }^{45}$ P. Vazquez Regueiro, ${ }^{44}$ C. Vázquez Sierra, ${ }^{30}$ S. Vecchi, ${ }^{19}$ J. J. Velthuis, ${ }^{51}$ M. Veltri, ${ }^{20, \text { aa }}$ A. Venkateswaran, ${ }^{65}$ M. Vernet,${ }^{8}$ M. Veronesi, ${ }^{30}$ M. Vesterinen, ${ }^{53}$ J. V. Viana Barbosa ${ }^{45}$ D. Vieira, ${ }^{4}$ M. Vieites Diaz, ${ }^{46}$ H. Viemann, ${ }^{71}$ X. Vilasis-Cardona, ${ }^{43, i}$ A. Vitkovskiy, ${ }^{30}$ V. Volkov, ${ }^{38}$ A. Vollhardt, ${ }^{47}$ D. Vom Bruch, ${ }^{11}$ B. Voneki, ${ }^{45}$ A. Vorobyev, ${ }^{36}$ V. Vorobyev, ${ }^{41, f}$ N. Voropaev,${ }^{36}$ R. Waldi, ${ }^{71}$ J. Walsh, ${ }^{27}$ J. Wang, ${ }^{3}$ J. Wang, ${ }^{5}$ 
M. Wang, ${ }^{3}$ Y. Wang, ${ }^{6}$ Z. Wang, ${ }^{47}$ D. R. Ward, ${ }^{52}$ H. M. Wark, ${ }^{57}$ N. K. Watson, ${ }^{50}$ D. Websdale, ${ }^{58}$ A. Weiden, ${ }^{47}$ C. Weisser, ${ }^{61}$ B. D. C. Westhenry, ${ }^{51}$ D. J. White, ${ }^{59}$ M. Whitehead, ${ }^{12}$ D. Wiedner, ${ }^{13}$ G. Wilkinson, ${ }^{60}$ M. Wilkinson, ${ }^{65}$ I. Williams, $^{52}$ M. Williams, ${ }^{61}$ M. R. J. Williams, ${ }^{59}$ T. Williams, ${ }^{50}$ F. F. Wilson, ${ }^{54}$ M. Winn, ${ }^{10}$ W. Wislicki, ${ }^{34}$ M. Witek, ${ }^{32}$ G. Wormser, ${ }^{10}$ S. A. Wotton, ${ }^{52}$ H. Wu, ${ }^{65}$ K. Wyllie, ${ }^{45}$ Z. Xiang, ${ }^{4}$ D. Xiao, ${ }^{6}$ Y. Xie, ${ }^{6}$ H. Xing, ${ }^{68}$ A. Xu, ${ }^{3}$ L. Xu, ${ }^{3}$ M. Xu, ${ }^{6}$ Q. Xu, ${ }^{4}$ Z. Xu, ${ }^{7}$ Z. Xu, ${ }^{3}$ Z. Yang, ${ }^{3}$ Z. Yang, ${ }^{63}$ Y. Yao, ${ }^{65}$ L. E. Yeomans, ${ }^{57}$ H. Yin, ${ }^{6}$ J. Yu, ${ }^{6, b b}$ X. Yuan, ${ }^{65}$ O. Yushchenko, ${ }^{42}$ K. A. Zarebski, ${ }^{50}$ M. Zavertyaev, ${ }^{1, w}$ M. Zdybal, ${ }^{32}$ M. Zeng, ${ }^{3}$ D. Zhang, ${ }^{6}$ L. Zhang, ${ }^{3}$ S. Zhang, ${ }^{3}$ W. C. Zhang, ${ }^{3, c c}$ Y. Zhang, ${ }^{45}$ A. Zhelezov, ${ }^{15}$ Y. Zheng, ${ }^{4}$ X. Zhou, ${ }^{4}$ Y. Zhou, ${ }^{4}$ X. Zhu, ${ }^{3}$ V. Zhukov, ${ }^{12,38}$ J. B. Zonneveld, ${ }^{55}$ and S. Zucchelli ${ }^{18, d}$

\title{
(LHCb Collaboration)
}

\author{
${ }^{1}$ Centro Brasileiro de Pesquisas Físicas $(C B P F)$, Rio de Janeiro, Brazil \\ ${ }^{2}$ Universidade Federal do Rio de Janeiro (UFRJ), Rio de Janeiro, Brazil \\ ${ }^{3}$ Center for High Energy Physics, Tsinghua University, Beijing, China \\ ${ }^{4}$ University of Chinese Academy of Sciences, Beijing, China \\ ${ }^{5}$ Institute Of High Energy Physics (IHEP), Beijing, China \\ ${ }^{6}$ Institute of Particle Physics, Central China Normal University, Wuhan, Hubei, China \\ ${ }^{7}$ Univ. Grenoble Alpes, Univ. Savoie Mont Blanc, CNRS, IN2P3-LAPP, Annecy, France \\ ${ }^{8}$ Université Clermont Auvergne, CNRS/IN2P3, LPC, Clermont-Ferrand, France \\ ${ }^{9}$ Aix Marseille Univ, CNRS/IN2P3, CPPM, Marseille, France \\ ${ }^{10}$ LAL, Univ. Paris-Sud, CNRS/IN2P3, Université Paris-Saclay, Orsay, France \\ ${ }^{11}$ LPNHE, Sorbonne Université, Paris Diderot Sorbonne Paris Cité, CNRS/IN2P3, Paris, France \\ ${ }^{12}$ I. Physikalisches Institut, RWTH Aachen University, Aachen, Germany \\ ${ }^{13}$ Fakultät Physik, Technische Universität Dortmund, Dortmund, Germany \\ ${ }^{14}$ Max-Planck-Institut für Kernphysik (MPIK), Heidelberg, Germany \\ ${ }^{15}$ Physikalisches Institut, Ruprecht-Karls-Universität Heidelberg, Heidelberg, Germany \\ ${ }^{16}$ School of Physics, University College Dublin, Dublin, Ireland \\ ${ }^{17}$ INFN Sezione di Bari, Bari, Italy \\ ${ }^{18}$ INFN Sezione di Bologna, Bologna, Italy \\ ${ }^{19}$ INFN Sezione di Ferrara, Ferrara, Italy \\ ${ }^{20}$ INFN Sezione di Firenze, Firenze, Italy \\ ${ }^{21}$ INFN Laboratori Nazionali di Frascati, Frascati, Italy \\ ${ }^{22}$ INFN Sezione di Genova, Genova, Italy \\ ${ }^{23}$ INFN Sezione di Milano-Bicocca, Milano, Italy \\ ${ }^{24}$ INFN Sezione di Milano, Milano, Italy \\ ${ }^{25}$ INFN Sezione di Cagliari, Monserrato, Italy \\ ${ }^{26}$ INFN Sezione di Padova, Padova, Italy \\ ${ }^{27}$ INFN Sezione di Pisa, Pisa, Italy \\ ${ }^{28}$ INFN Sezione di Roma Tor Vergata, Roma, Italy \\ ${ }^{29}$ INFN Sezione di Roma La Sapienza, Roma, Italy \\ ${ }^{30}$ Nikhef National Institute for Subatomic Physics, Amsterdam, Netherlands \\ ${ }^{31}$ Nikhef National Institute for Subatomic Physics and VU University Amsterdam, Amsterdam, Netherlands \\ ${ }^{32}$ Henryk Niewodniczanski Institute of Nuclear Physics Polish Academy of Sciences, Kraków, Poland \\ ${ }^{33}$ AGH-University of Science and Technology, Faculty of Physics and Applied Computer Science, \\ Kraków, Poland \\ ${ }^{34}$ National Center for Nuclear Research (NCBJ), Warsaw, Poland \\ ${ }^{35}$ Horia Hulubei National Institute of Physics and Nuclear Engineering, Bucharest-Magurele, Romania \\ ${ }^{36}$ Petersburg Nuclear Physics Institute NRC Kurchatov Institute (PNPI NRC KI), Gatchina, Russia \\ ${ }^{37}$ Institute of Theoretical and Experimental Physics NRC Kurchatov Institute (ITEP NRC KI), Moscow, \\ Russia, Moscow, Russia \\ ${ }^{38}$ Institute of Nuclear Physics, Moscow State University (SINP MSU), Moscow, Russia \\ ${ }^{39}$ Institute for Nuclear Research of the Russian Academy of Sciences (INR RAS), Moscow, Russia \\ ${ }^{40}$ Yandex School of Data Analysis, Moscow, Russia \\ ${ }^{41}$ Budker Institute of Nuclear Physics (SB RAS), Novosibirsk, Russia \\ ${ }^{42}$ Institute for High Energy Physics NRC Kurchatov Institute (IHEP NRC KI), \\ Protvino, Russia, Protvino, Russia \\ ${ }^{43}$ ICCUB, Universitat de Barcelona, Barcelona, Spain \\ ${ }^{44}$ Instituto Galego de Física de Altas Enerxías (IGFAE), Universidade de Santiago de Compostela, \\ Santiago de Compostela, Spain
}


${ }^{45}$ European Organization for Nuclear Research (CERN), Geneva, Switzerland

${ }^{46}$ Institute of Physics, Ecole Polytechnique Fédérale de Lausanne (EPFL), Lausanne, Switzerland

${ }^{47}$ Physik-Institut, Universität Zürich, Zürich, Switzerland

${ }^{48}$ NSC Kharkiv Institute of Physics and Technology (NSC KIPT), Kharkiv, Ukraine

${ }^{49}$ Institute for Nuclear Research of the National Academy of Sciences (KINR), Kyiv, Ukraine

${ }^{50}$ University of Birmingham, Birmingham, United Kingdom

${ }^{51}$ H.H. Wills Physics Laboratory, University of Bristol, Bristol, United Kingdom

${ }^{52}$ Cavendish Laboratory, University of Cambridge, Cambridge, United Kingdom

${ }^{53}$ Department of Physics, University of Warwick, Coventry, United Kingdom

${ }^{54}$ STFC Rutherford Appleton Laboratory, Didcot, United Kingdom

${ }^{55}$ School of Physics and Astronomy, University of Edinburgh, Edinburgh, United Kingdom

${ }^{56}$ School of Physics and Astronomy, University of Glasgow, Glasgow, United Kingdom

${ }^{57}$ Oliver Lodge Laboratory, University of Liverpool, Liverpool, United Kingdom

${ }^{58}$ Imperial College London, London, United Kingdom

${ }^{59}$ Department of Physics and Astronomy, University of Manchester, Manchester, United Kingdom

${ }^{60}$ Department of Physics, University of Oxford, Oxford, United Kingdom

${ }^{61}$ Massachusetts Institute of Technology, Cambridge, Massachusetts, USA

${ }^{62}$ University of Cincinnati, Cincinnati, Ohio, USA

${ }^{63}$ University of Maryland, College Park, Maryland, USA

${ }^{64}$ Los Alamos National Laboratory (LANL), Los Alamos, USA

${ }^{65}$ Syracuse University, Syracuse, New York, USA

${ }^{66}$ Laboratory of Mathematical and Subatomic Physics, Constantine,

Algeria [associated with Universidade Federal do Rio de Janeiro (UFRJ), Rio de Janeiro, Brazil]

${ }^{67}$ Pontifícia Universidade Católica do Rio de Janeiro (PUC-Rio), Rio de Janeiro, Brazil [associated with

Universidade Federal do Rio de Janeiro (UFRJ), Rio de Janeiro, Brazil]

${ }^{68}$ South China Normal University, Guangzhou, China (associated with Center for High Energy Physics, Tsinghua University, Beijing, China)

${ }^{69}$ School of Physics and Technology, Wuhan University, Wuhan, China (associated with Center for High Energy Physics, Tsinghua University, Beijing, China)

${ }^{70}$ Departamento de Fisica, Universidad Nacional de Colombia, Bogota, Colombia (associated with LPNHE, Sorbonne Université, Paris Diderot Sorbonne Paris Cité, CNRS/IN2P3, Paris, France)

${ }^{71}$ Institut für Physik, Universität Rostock, Rostock, Germany (associated with Physikalisches Institut, Ruprecht-Karls-Universität Heidelberg, Heidelberg, Germany)

${ }^{72}$ Van Swinderen Institute, University of Groningen, Groningen, Netherlands (associated with Nikhef National Institute for Subatomic Physics, Amsterdam, Netherlands)

${ }^{73}$ National Research Centre Kurchatov Institute, Moscow, Russia [associated with Institute of Theoretical and Experimental Physics NRC Kurchatov Institute (ITEP NRC KI), Moscow, Russia, Moscow, Russia]

${ }^{74}$ National University of Science and Technology "MISIS", Moscow, Russia [associated with Institute of Theoretical and Experimental Physics NRC Kurchatov Institute (ITEP NRC KI),

Moscow, Russia, Moscow, Russia]

${ }^{75}$ National Research University Higher School of Economics, Moscow, Russia (associated with Yandex School of Data Analysis, Moscow, Russia)

${ }^{76}$ National Research Tomsk Polytechnic University, Tomsk, Russia [associated with Institute of Theoretical and Experimental Physics NRC Kurchatov Institute (ITEP NRC KI), Moscow, Russia, Moscow, Russia]

${ }^{77}$ Instituto de Fisica Corpuscular, Centro Mixto Universidad de Valencia-CSIC, Valencia, Spain (associated with ICCUB, Universitat de Barcelona, Barcelona, Spain)

${ }^{78}$ University of Michigan, Ann Arbor, USA (associated with Syracuse University, Syracuse, New York, USA)

†antimo.palano@ba.infn.it

${ }^{\mathrm{a}}$ Deceased.

${ }^{\mathrm{b}}$ Also at Laboratoire Leprince-Ringuet, Palaiseau, France.

${ }^{\mathrm{c}}$ Also at Università di Genova, Genova, Italy.

${ }^{\mathrm{d}}$ Also at Università di Bologna, Bologna, Italy.

${ }^{\mathrm{e}}$ Also at Università di Modena e Reggio Emilia, Modena, Italy.

${ }^{\mathrm{f}}$ Also at Novosibirsk State University, Novosibirsk, Russia.

${ }^{\mathrm{g}}$ Also at Università di Ferrara, Ferrara, Italy.

${ }^{\mathrm{h}}$ Also at Università di Milano Bicocca, Milano, Italy.

${ }^{\mathrm{i}}$ Also at LIFAELS, La Salle, Universitat Ramon Llull, Barcelona, Spain.

${ }^{\mathrm{j}}$ Also at Università di Pisa, Pisa, Italy. 
${ }^{\mathrm{k}}$ Also at Università di Bari, Bari, Italy.

${ }^{1}$ Also at INFN Sezione di Trieste, Trieste, Italy.

${ }^{\mathrm{m}}$ Also at Università degli Studi di Milano, Milano, Italy.

${ }^{\mathrm{n}}$ Also at Universidade Federal do Triângulo Mineiro (UFTM), Uberaba-MG, Brazil.

${ }^{\circ}$ Also at AGH-University of Science and Technology, Faculty of Computer Science, Electronics and Telecommunications, Kraków, Poland.

${ }^{\mathrm{p}}$ Also at Università di Siena, Siena, Italy.

${ }^{\mathrm{q}}$ Also at Lanzhou University, Lanzhou, China.

${ }^{\mathrm{r}}$ Also at Università di Padova, Padova, Italy.

${ }^{\mathrm{s}}$ Also at Scuola Normale Superiore, Pisa, Italy.

${ }^{\mathrm{t}}$ Also at Università di Cagliari, Cagliari, Italy.

${ }^{\mathrm{u}}$ Also at MSU-Iligan Institute of Technology (MSU-IIT), Iligan, Philippines.

${ }^{v}$ Also at Hanoi University of Science, Hanoi, Vietnam.

${ }^{w}$ Also at P.N. Lebedev Physical Institute, Russian Academy of Science (LPI RAS), Moscow, Russia.

${ }^{\mathrm{x}}$ Also at Università di Roma Tor Vergata, Roma, Italy.

${ }^{\mathrm{y}}$ Also at Università della Basilicata, Potenza, Italy.

${ }^{\mathrm{z}}$ Also at Università di Roma La Sapienza, Roma, Italy.

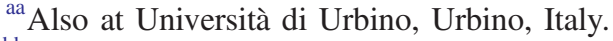

${ }^{\mathrm{bb}}$ Also at Physics and Micro Electronic College, Hunan University, Changsha City, China.

${ }^{\mathrm{cc}}$ Also at School of Physics and Information Technology, Shaanxi Normal University (SNNU), Xi'an, China. 